\title{
¿La región como trampolín para la emergencia global? Las estrategias regionales de Brasil y Turquía
}

\author{
Sebastien Adins*
}

\begin{abstract}
RESUMEN
Tomando en consideración el carácter transitorio del actual sistema internacional, el presente artículo pretende analizar la relación entre la emergencia global de diversas potencias del Sur y su proyección regional. Luego de una exploración de la literatura académica sobre las potencias regionales, medias y emergentes, se realiza una comparación entre las estrategias regionales de dos Estados, Brasil y Turquía, basada en cuatro dimensiones: sus recursos (relativos) de poder, la autopercepción respecto a la región, las estrategias y roles propios de su política regional y la recepción de dicha proyección por los vecinos y las principales potencias extrarregionales. A partir de esta comparación, se determina de qué manera estas estrategias regionales se relacionan con la aspiración de emergencia global de ambas potencias.
\end{abstract}

Palabras clave: potencias regionales, potencias emergentes, jerarquía de poder, roles de política exterior, tesis del trampolín, Brasil, Turquía

The region as a springboard for global emergency? The regional strategies of Brazil and Turkey

\section{Abstract}

Taking into consideration the transitory nature of the current international system, this article aims to analyze the relationship between the global emergence of several powers from the South and their regional policies. After an exploration of the academic literature on regional, middle and rising powers, the author compares the regional strategies of two states, Brazil and Turkey, based on four dimensions: their relative power resources, the self-

\footnotetext{
* Magíster en Relaciones Internacionales de la Universidad de Gante (Bélgica) y doctor en Ciencia Política de la Pontificia Universidad Católica del Perú. Profesor e investigador en la Pontificia Universidad Católica del Perú y la Academia Diplomática del Perú. Autor de diversas publicaciones sobre relaciones internacionales. Correo electrónico: sadins@pucp.pe
}

iD https://orcid.org/0000-0002-7029-3369 
perception regarding the region, the strategies and roles that constitute their regional policy and the reception of this projection by its neighbors and the main extra-regional powers. From this comparison, the relationship will be established between the regional strategies of both countries and their global emergence aspirations.

Keywords: regional powers, rising powers, power hierarchy, foreign policy roles, springboard hypothesis, Brazil, Turkey

\section{Introducción}

A partir de la década pasada se hizo popular la tesis sobre la transición del orden internacional hacia una configuración multipolar, crecientemente post occidental y con un notable rol para las potencias emergentes y regionales. Respecto a la relación entre ambas potencias, varios académicos y políticos dieron a entender que la emergencia hacia el estatus de gran potencia necesariamente tenía que pasar por el de potencia regional. Es decir, según esta "tesis del trampolín», más allá del reconocimiento mundial que implica dicho proceso, los vecinos son críticos para coadyuvar a la emergencia global.

Luego de realizar una revisión de la literatura sobre jerarquías de poder y potencias medias, emergentes y regionales, este artículo tiene como objetivo determinar los instrumentos y el alcance de los proyectos regionales en marcha desde inicios del presente siglo, de dos potencias medias emergentes: Brasil y Turquía. Luego se evalúa en qué medida las estrategias regionales de los dos países han contribuido con su emergencia global y por qué razones ambas han entrado en crisis en estos últimos años. Como tal, se postula que el estatus de potencia regional implica el cumplimiento con ciertos roles hacia los vecinos, lo que absorbe cuantiosos recursos — aún escasos para una potencia media-, generando así una tensión entre ambas misiones (la regional y global). Además, está claro que las grandes potencias consolidadas solo se muestran dispuestas a reconocer la emergencia de las potencias medias bajo ciertas condiciones.

\section{Marco conceptual y teórico}

Iniciamos el presente artículo con una exploración de los diversos enfoques existentes en la literatura sobre las potencias medias, emergentes y regionales, así como las estrategias implementadas por estas últimas. El objetivo de este marco conceptual consiste en marcar las diferencias y la relación entre los tres tipos de potencias mencionados, así como elaborar las variables que nos servirán como base de comparación entre nuestros dos casos. 
Antes de pasar al marco conceptual propiamente dicho, caben tres comentarios como puntos de partida de la investigación. Primero, al momento de hablar sobre las potencias se toca directamente uno de los conceptos clave de la ciencia política: el poder. Pese a las diversas perspectivas que existen para analizar dicho fenómeno multidimensional, se distinguen dos principales, conocidas como el poder acumulativo y el poder relacional. Mientras que el primer enfoque considera al «actor poderoso» meramente como rico en determinados recursos, el segundo lo considera como el efecto de una situación y/o por la influencia que dicho poder genera en otros actores (Vallès, 2010). De la misma manera, en las relaciones internacionales se suele distinguir entre el poder potencial (o «poder base», conformado por las capacidades estructurales) y el poder real de un actor.

En línea con lo señalado por Raymond Aron, Celestino del Arenal (1983) considera que el nexo entre ambas dimensiones lo constituye la movilización del poder. Este proceso de conversión a su vez resulta, principalmente, del contexto (el sistema internacional que interfiere, por ejemplo, en la fungibilidad de los recursos) y la voluntad misma del actor, con sus respectivos objetivos. Por ello, consideramos que una potencia no solamente debe mostrar una preponderancia material, sino además generar algún impacto en otros Estados del sistema global/regional y obtener el reconocimiento internacional como tal.

Segundo, consideramos que coexisten varias jerarquías de poder en el sistema internacional que se sobreponen mutuamente. En este sentido distinguimos entre la jerarquía global, las jerarquías regionales y las jerarquías sectoriales. En el primer nivel caben la potencia hegemónica, una serie de grandes potencias, potencias medias y las menores. A su vez, las jerarquías regionales cuentan con una (en el caso de un orden regional unipolar) o varias (cuando el orden regional es de carácter bipolar o multipolar) gran(des) potencia(s) regional(es), acompañada(s) de potencias regionales secundarias. Por último, algunas potencias medias o pequeñas pueden figurar como líderes en algún nicho de la diplomacia, convirtiéndose así en "potencias de nicho» (Cooper, 2013).

Tercero, coincidimos con los postulados de Buzan y Waever (2004) sobre la trayectoria del orden internacional desde una configuración «2+3» durante la era de la Guerra Fría (Estados Unidos y la Unión Soviética como los únicos dos «superpotencias» y las tres grandes potencias de la época: China, Japón y Alemania); pasando por el «momento unipolar» $\mathrm{o}$ " $1+4$ » (donde Rusia pasó a ser una "gran potencia») al actual esquema « $0+\mathrm{X}$ », un orden sin superpotencias pero con un grupo de "grandes potencias» —actualmente: Estados Unidos, China, Rusia, la Unión Europea - UE (o en su reemplazo el «condominio» Alemania-Francia) y Japón y, posiblemente en unas décadas más, India y Brasil-. 


\subsection{Las potencias medias}

El concepto de potencias medias continúa siendo una categoría contestada en la literatura sobre jerarquía de poder. Según Carsten Holbraad (1972), el término (en su versión alemana, Mittelmacht) surgió por primera vez a mediados del siglo XIX pero aglutinaba varios significados en sí: más allá de ser un Estado equipado con una fuerza media, también podía referirse a una potencia (geográficamente) céntrica y equilibradora del sistema; una potencia mediadora en conflictos; y hasta un "puente» entre civilizaciones. En la misma línea, según Cooper (2011), actualmente se puede distinguir entre tres perspectivas sobre las potencias medias.

Primero existe la lectura realista que considera a las potencias medias como aquellos Estados con ciertos atributos nacionales de rango medio, sobre todo a nivel económico (PBI), demográfico o militar, es decir, las que están ubicadas justamente por debajo de las grandes potencias del sistema internacional. Así, por ejemplo, se podría tildar como potencias medias a los miembros del G20 de países industrializados y emergentes que (aún) no cuentan con los recursos de una gran potencia. Por ello, países como Brasil, Corea del Sur, España, Italia, México, Sudáfrica o Turquía serían ejemplos del grupo. Fue además desde esta perspectiva que Wight (1978) elaboró la siguiente definición de una potencia media:

A middle power is a power with such a military strength, resources and strategic position that in peacetime the great powers bid for its support, and in wartime, while it has no hope of winning a war against a great power, it can hope to inflict costs on a great power out of proportion to what the great power can hope to gain by attacking it. (p. 65)

Desde una segunda perspectiva se le considera a la potencia media a partir del rol o estilo de diplomacia (middle power statecraft) en el sistema internacional. Así, en el contexto de la Guerra Fría, algunos Estados — tales como los países nórdicos o Canadá- empezaron a implementar una política exterior normativa y dirigida hacia el multilateralismo (principalmente mediante la construcción de alianzas o desde organizaciones internacionales), la cooperación, la mediación ante conflictos, el respeto por el derecho internacional y el fortalecimiento de los regímenes internacionales.

Al enfatizar mecanismos de convicción e incentivos —en detrimento de la coerción-, la proyección de poder blando ${ }^{1}$ y la defensa del interés general encima del nacional, dichas potencias medias han sido consideradas como una especie de «buenos

\footnotetext{
1 El top-15 para 2018 del Índice Soft Power 30, lo ocupan las cinco principales potencias occidentales (en este orden: Reino Unido, Francia, Alemania, Estados Unidos y Japón), seguidas por diez potencias medias tradicionales (Canadá, Suiza, Suecia, Países Bajos, Australia, Dinamarca, Italia, Noruega, España y Finlandia), manifestando claramente el sesgo occidental de dichos estudios.
} 
ciudadanos» del sistema (van der Westhuizen, 1998). De esta manera también, Cox y Sinclair (1996) postularon lo siguiente sobre el concepto:

The middle power is likely to be in the middle rank of material capabilities, but it also stands in the middle of situations of conflict. It seeks to expand the area of common ground, which will make it possible to curtail risk in the management of conflict. Possessing middle range capability (military and economic) is a necessary condition of the ability to play this role, but it is not an adequate predictor of a disposition to play it. An ability to stand a certain distance from direct involvement in major conflicts, a sufficient degree of autonomy in relation to major powers, a commitment to orderliness and security in interstate relations and to the facilitation of orderly change in the world system are the critical elements for the fulfillment of the middle power role. (p. 244)

No obstante, el problema de este segundo enfoque es que apenas existe una correlación entre el tamaño de un Estado, y como tal su posición en la jerarquía internacional, y la conducta de su política exterior (Cooper, 2011). Así, por ejemplo, mientras que la proyección externa de Japón durante la Guerra Fría coincidió con la de una potencia media, a nivel de capacidades materiales el país asiático claramente superó dicha categoría. De la misma manera, países como Noruega o Suiza, con cuerpos diplomáticos profesionales y líderes de algunas iniciativas diplomáticas, no agrupan la suficiente cantidad de recursos para poder ser clasificados como potencias medias.

Por último, Cooper (2013) considera que algunos Estados —entre otros; Australia, Canadá y Suecia - no solamente califican como potencias medias a nivel estructural o por su comportamiento internacional, sino que además se identifican como tal (por ello, el término self-identified middle powers) ${ }^{2}$. Esta tercera perspectiva sobre las potencias medias coincide mayormente con el paradigma constructivista de Relaciones Internacionales al enfatizar el carácter ideacional de la categoría y los intereses que sustentan dicha identidad. En otros términos, la idea de "potencia media» estaría construida y cultivada por los arquitectos de política exterior de un grupo reducido de Estados, a fin de satisfacer los intereses nacionales y aumentar el status de su país en el escenario internacional. Así también lo estipula Janis van der Westhuizen (1998):

Middle powers are not simply middle powers because of their participation in mediation, bridge-building activity or cooperation in certain technical areas, but because it suits their long term interest vis-à-vis world order, the world economy, and the pursuit of dominant societal values and interests, all supported by significant material/technical/bureaucratic capabilities to do so. (p. 439)

\footnotetext{
2 En realidad, las primeras manifestaciones del uso moderno del término middle power se da en los años 1944 y 1945 cuando tanto Canadá como Australia empezaron a autoatribuirse dicho rol (Robertson, 2017, p. 357).
} 
Es decir, ante la falta relativa de recursos frente a las grandes potencias, las potencias medias claramente tienen un interés para actuar desde un marco multilateral o alianzas internacionales.

Si durante la Guerra Fría el concepto de las potencias medias estuvo asociado mayormente con países pertenecientes al Gran Occidente, con economías desarrolladas (y relativamente iguales) y sistemas políticos democráticos y estables, esto empezó a cambiar con el surgimiento a fines del siglo pasado de una «segunda generación de potencias medias", conocidas como emerging middle powers (a partir de aquí: PME). Según Jordaan (2003), contrariamente a las potencias medias tradicionales, las PME generalmente provienen del Sur (de modo más específico, de la semi-periferia del sistema económico global) y, pese a que comparten varios atributos con las potencias medias tradicionales, difieren de las últimas en al menos tres aspectos.

Primero, respecto al estilo de política exterior, suelen adoptar una política exterior basada en intervenciones denominadas "heroicas», entendidas como acciones emprendidas por el líder político de un Estado a fin de aumentar el perfil internacional de su país (Jordaan, 2003). Este estilo de política exterior, contrariamente a las «intervenciones rutinarias» — más propias de las potencias medias tradicionales—, resulta tanto de la escasez relativa de recursos disponibles, como de la falta de legitimidad interna de los gobiernos.

Segundo, al considerar su posición en el sistema económico internacional, las PME generalmente se ponen a favor de una política reformista —-más no revisionista— ${ }^{3}$, en el terreno económico. Esta proyección se manifiesta a través de sus llamados a favor de una redistribución de las estructuras de poder y riqueza — por ejemplo, mediante un multilateralismo más inclusivo- y de un mayor reconocimiento de los reclamos del Sur global en la sociedad internacional (Nel, 2010). Mientras tanto, las potencias medias tradicionales se caracterizaron/caracterizan por sus políticas, según Jordaan (2003), de «apaciguamiento» y hasta de legitimización del orden internacional y de las políticas hegemónicas.

Tercero, más que ejercer algún tipo de liderazgo funcional en la agenda global, las PME cuentan con una política explícitamente regional, básicamente vinculada

\footnotetext{
3 Consideramos que tanto en dossiers económicos como políticos, las potencias pueden asumir tres posibles orientaciones: por un lado, la defensa del statu-quo; en el otro extremo la propuesta, más radical, del revisionismo; y una postura intermedia que refiere al reformismo. Tal como lo postularon Kugler y Organski (1989), dicha postura depende mayormente del grado de «satisfacción» de la potencia en cuestión frente al sistema internacional existente (y sus respectivos arreglos institucionales, su división territorial, su distribución económica, etc...) y el prestigio que goza en él. De esta manera, por ejemplo países como Alemania, Japón o Canadá pertenecerían al primer grupo; en el segundo cabrían Rusia, Irán y Venezuela; mientras que Brasil, India y Turquía serían ejemplos de potencias de orientación reformista.
} 
a los ámbitos del comercio y la gobernanza de seguridad regional. Esta «vocación regional» más ausente entre las potencias medias tradicionales, resulta principalmente de su predominio material dentro de su región (siendo los ejemplos clásicos Brasil y Sudáfrica). Además, está claro que la proyección regional de las PME — por ejemplo, mediante la creación de algún organismo de gobernanza regional一, puede contribuir con la internacionalización de sus empresas (en búsqueda de mercados o recursos en países vecinos), la estabilización de su entorno geopolítico, y en general, puede elevar la capacidad de negociación, así como su legitimidad a nivel global.

Por una combinación de las políticas unilaterales de Estados Unidos en la era Bush (en detrimento del multilateralismo), indicios de una regionalización del sistema post-Guerra Fría y el empoderamiento económico de varias economías del Sur, las clásicas potencias medias claramente han perdido influencia a favor de las de segunda generación (Dewitt y Christie, 2007). Fue precisamente en este contexto que se percibió una creciente atención académica a favor de las potencias emergentes y regionales.

\subsection{Las potencias emergentes}

De la misma manera como las otras categorías de potencias, la de «potencias emergentes» (rising powers ${ }^{4}$ ) suele ser altamente ambigua; para empezar, por su origen y connotación principalmente económica. Así, en el año 1981, Antoine van Agtmael, funcionario de la Corporación Financiera Internacional, estrenaría el concepto «mercado emergente» aludiendo a aquellas economías en vías de desarrollo inmersas en la restructuración de su deuda externa (y con un mercado de valores prometedor) ${ }^{5}$.

Luego, a partir de la década pasada, el concepto estuvo estrechamente asociado con las diversas siglas que se acuñaron desde la banca internacional para referirse a las economías del Sur con mayor potencial (entre otras; los BRIC, Next 11, MIKTA y CIVETS). El hype sobre las potencias emergentes de estos ańos claramente coincidió con la erosión del «momento unipolar», la narrativa sobre un incipiente orden posamericano (Zakaria, 2008 y Acharya, 2018) o posoccidental (Serfaty, 2011 y Stuenkel, 2016), así como el diferente impacto que generó la crisis financiera de 2008 en el Norte y Sur.

\footnotetext{
4 Algunos, como Acharya, distinguen entre los rising y los emerging powers, así: «[...] the term "rising powers» is normally associated with countries that have a clear potential to become great powers, such as China, India, and Brazil. The term «emerging powers» indicates countries such as Indonesia, South Korea, Mexico, Nigeria, and South Africa, which are not perceived to be headed for international great-power status» (2018, p. 77).

5 Luego, en 1988, el ponderador de fondos de capital de inversión MSCI empezó con la publicación de un índice anual de mercados emergentes. Si dicho índice inicialmente contara con diez países, luego de treinta años ya agrupa a veinticuatro países. Véase: https://www.msci.com/emerging-markets
} 
Finalmente, con una connotación más política, en 2004, el Consejo Nacional de Inteligencia de EE.UU. se refirió a China, India, Brasil, Indonesia, Rusia y Sudáfrica en cuanto "potencias emergentes» (rising powers), reemplazando así el término de «estados pivote» (pivotal states), muy común en la década de 1990 (National Intelligence Councial [NIC], 2004) ${ }^{6}$.

Desde una perspectiva más propia de Relaciones Internacionales, Fonseca, Paes y Cunha definen una potencia emergente como: «a country with a set of material and ideational attributes along with a specific political behavior, a reformist or revisionist activism in international order» (2016, p. 61), apuntando con lo último que la emergencia de ciertos Estados implica un aumento del grado de autonomía en la formulación de su política exterior. Además, para los investigadores brasileños, la emergencia de potencias constituye el resultado de una transformación doméstica que impacta al sistema, así como de una estrategia internacional determinada. Por un lado, a nivel doméstico, las potencias emergen a raíz de una mejora en la posición de la división internacional del trabajo (entendida como más cercana al «centro» del sistema económico global), originando así una redistribución de la riqueza global, así como de la conversión de poder latente en capacidad militar, sobre todo en términos comparativos con los demás miembros de su región. Por otro lado, la estrategia internacional de las potencias emergentes suele ser del tipo institucional. Esto se expresa, según los autores, a nivel regional, con la búsqueda de una hegemonía, basada en un proyecto de liderazgo político y/o económico, mientras que predomina, a nivel global, el activismo institucional a fin de moldear un orden normativo más favorable a su emergencia como potencia (2016, p. 61).

A su vez, Amrita Narkilar define a las potencias emergentes como «those states that have established themselves as veto-players in the international system, but have not acquired agenda-setting power» (2013, pp. 561-562). Para la autora, dicha emergencia no solamente resulta del crecimiento económico o militar del Estado en cuestión, sino, mayormente, del proceso de negociación que la acompaña: «The rise of new powers is seldom solely a function of growing economic or military prowess; much depends on how power is exercised, in relation to whom, the motivations that underpin this exercise, and further how action and reaction are interpreted and misinterpreted. The rise of new powers is thus fundamentally a story of bargaining and negotiation» (2013, p. 561). Entre las estrategias más usadas por los emergentes encontramos al soft balancing frente a las potencias consolidadas; el establecimiento

\footnotetext{
6 Chase, Hill y Kennedy (1999) definieron a un Estado pivote como «a key country whose future may not only determine the success of failure of its region but also significantly affect international stability" (Nolte, 2006, p. 7). Mientras, según el reporte del NCI, las potencias emergentes se caracterizaron por un crecimiento económico alto, capacidades militares en expansión, promoción activa de altas tecnologías y poblaciones largas (NIC, 2004).
} 
de coaliciones, tanto de alcance regional como mediante alianzas Sur-Sur; y el enmarcado de sus demandas en términos que apelan a principios de justicia (Narkilar, 2013, p. 561; Kahler, 2013 y Hurrell, 2006).

La mayoría de investigaciones sobre el tema omite la cuestión respecto a qué atributos exactamente necesita presentar un Estado para ser clasificado como potencia emergente o cuando dicha emergencia exactamente culmina en el ingreso al concierto de grandes potencias. No obstante, sí parece estar claro que, más allá de los recursos de poder y el ya mencionado proceso de negociación permanente con las demás potencias, el ser potencia constituye un estatus en el sistema internacional, tal como lo postula Hurrell (2006):

Being a great power has never been solely about the possession of large amounts of crude material power. It has been closely related to notions of legitimacy and authority. A state can claim great power status, but membership of the club of great powers us a social category that depends on recognition by others: by your peers in the club, but also by smaller and weaker states willing to accept the legitimacy and authority of those at the top of the international hierarchy. (p. 4)

De esta manera, el estatus de (gran) potencia no solo dependería del reconocimiento por parte de las demás potencias, sino también de potencias menores, principalmente aquellas ubicadas en la región.

\subsection{Las potencias regionales}

Mientras que los conceptos de potencias medias y emergentes están conectados con el orden internacional (global), las potencias regionales se insertan en la jerarquía de los (sub-) sistemas regionales. Al respecto, desde la década de 1990, se hizo popular la lectura sobre una creciente "regionalización», paralelamente al proceso de globalización, de la política internacional. Así, Hurrell (2007) postuló lo siguiente sobre el asunto:

For many analysts the end of the Cold War removed the security overlay that had either dominated, or at least strongly influenced, patterns of regional security in many parts of the world. Regions were "set free», and regional logics came to predominate both in the production of insecurity — both traditional and, especially, nontraditional [...] - and in the management of insecurity, with increased incentives for states within a region to deal with their own problems and a decreased incentive for outside powers to intervene or become involved (p. 128).

Este presunto proceso de regionalización de las agendas internacionales (principalmente la de seguridad y la comercial), en combinación con el impacto que generó el crecimiento económico diferencial, sostenido durante una década, de varias grandes economías en el Sur hizo que cada vez más la academia analizará el fenómeno de 
las potencias regionales (PR). Sin embargo, el término tampoco está exento de problemas, principalmente por tres razones.

En primer lugar, más allá del consenso sobre algunas de las características de las potencias regionales (entre otras, pertenencia a una región determinada, preponderancia de recursos de poder y capacidad para influir sobre asuntos regionales) ${ }^{7}$, no existe claridad sobre el alcance real de las mismas. En este sentido, Prys (2010) advierte que:

Empirical studies in fact often come to the conclusion that states described [as regional powers], generally appear less powerful than their factual material preponderance might suggest: they do not provide public goods, such as order and stability, nor do they have an extraordinary impact on the behavior of other states in their region. (p. 4)

Efectivamente, un país puede mostrar una superioridad relativa de recursos en una región determinada, pero dicho predominio puede quedar obsoleto ante la penetración de una región por parte de las potencias extrarregionales o ante las estrategias de balanceo de sus vecinos ${ }^{8}$. Por ello también, Garzón Pereira (2013, pp. 29-30) distingue entre dos posibles escenarios resultantes del surgimiento de las PR: un orden internacional multipolar que se basa en una serie de unipolaridades regionales —es decir: grandes potencias con sus respectivas zonas de influencia- o, un escenario probablemente más realista, un sistema caracterizado por el «multi-multipolarismo", donde a nivel global dominan una serie de grandes potencias que, en su propia región, quedan desafiadas por parte de potencias regionales secundarias ${ }^{9}$, con o sin el apoyo de potencias extrarregionales.

Por otro lado, cabe mencionar que en tiempos de globalización social y económica, la proyección regional de las PR no solamente resulta de las políticas de Estado, sino también de las acciones de actores no estatales, entre ellas las empresas multinacionales - principalmente, mediante sus inversiones en los países vecinos-o representantes de la sociedad civil. Efectivamente, tal como lo postula Mitchell (2016):

\footnotetext{
7 Así, por ejemplo, Østerud caracterizó a la PR (regional great power) como: a state which is (1) geographically part of the delineated region, (2) is able to stand up against any coalition of other states in the region, (3) is highly influential in regional affairs, and (4) contrary to a middle power, might also be a great power of the world scale in addition to its regional standing (Nolte, 2010).

8 Por otro lado, si la preponderancia de poder duro no es suficiente para ser tildado como PR, según un estudio de Lemke (2010), sí existe una correlación entre la proporción de poder de la PR en su región y el nivel de militarización, así como el número de organizaciones regionales en ella.

9 Por ello, Föhrig (2007) menciona la existencia de «actores de impugnación regional» que son aquellos [Estados] que - aún sin capacidad de vetar formalmente acciones de los estados pivote- deben brindar su consenso para que un actor regional alcance sus objetivos en el ámbito multilateral».
} 
"with the synchronization of ideology between the state and transnational capital, it becomes a critical tool in exercising hegemony or counter-hegemony» (p. 9).

Una segunda dificultad que enfrenta el concepto de PR concierne su relación ambigua con el término «liderazgo regional», entendido aquí como la capacidad y voluntad de un Estado para proveer bienes públicos a nivel regional y para guiar a sus vecinos (convertidos así en «seguidores») en la formulación y realización de objetivos comunes de política exterior. Dicho liderazgo se puede manifestar a través la formulación de una agenda regional (el llamado «liderazgo intelectual»), acciones de mediación y convocatoria del líder (el «liderazgo empresarial $»^{10}$ ) o la representación de los intereses de los demás vecinos en foros extrarregionales.

Mientras que algunos autores, como Nolte (2006), Schirm (2005) o Flemes (2007), consideran la pretensión de ejercer un papel de liderazgo regional como una característica esencial de cualquier PR, otros solo lo tildan como una de varias estrategias disponibles para una potencia regional. Dicho de otra manera, resulta erróneo suponer que las potencias regionales generarían, automáticamente, un liderazgo (benigno) sobre su vecindad. Así, por ejemplo, Destradi (2010) distingue entre tres estrategias regionales: el imperio (caracterizado por ser unilateral y coercitivo); la hegemonía (una forma de poder más sutil donde el interés propio del hegemón prima pero se presenta como si fuera un objetivo colectivo); y el liderazgo (donde el país dominante guía a un grupo de Estados a fin de realizar ciertos objetivos comunes). A su vez, Prys (2010) define al «hegemón regional» como un país regionalmente preponderante que se autopercibe y es reconocido/aceptado por terceros en cuanto "líder natural», influye en asuntos regionales mediante un proceso de socialización y además provee ciertos bienes públicos a los vecinos. Los dos «polos negativos» de la noción serían, aún según la autora, el imperio y el desapego (detachment).

Entre los drivers que explican la determinación por una de dichas estrategias se menciona la existencia de una amenaza externa (tanto de seguridad como económica), alguna demanda societal (por ejemplo, por parte de un grupo de interés), la proyección de una imagen positiva a fin de aumentar la legitimidad de la potencia, así como motivos neopatrimoniales (Hulse, 2016, pp. 12-14). Finalmente, según Flemes y Wojczewski (2011, pp. 10-13), el éxito del liderazgo ejercido por una potencia regional o dicho en otros términos, el seguimiento de la PR depende básicamente del grado de convergencia entre los intereses de los Estados, la proyección

\footnotetext{
10 Según Oran Young (1991), el liderazgo intelectual refiere a la generación de ideas influyentes para los partícipes de una negociación. En cambio, el liderazgo empresarial consiste en la posesión de ciertas habilidades de negociación, tales como la agenda setting, el desbloqueo de negociaciones o la presentación de argumentos sólidos. Finalmente, el liderazgo estructural implica la movilización de determinados recursos, tanto de índole material como simbólica.
} 
de ideas o normas que coinciden con las creencias de los potenciales «seguidores» («hegemonía consensual» en términos de Burges ${ }^{11}$ ), la provisión de ciertos incentivos materiales (como paymaster) y la disposición a compartir poder de modo permanente —o en términos de Pedersen (2002), de ejercer una "hegemonía cooperativa», por ejemplo mediante la creación de regímenes de gobernanza regional ${ }^{12}$ -

Tercero, no queda claro cómo se relacionan las políticas regionales y globales de las potencias regionales. En este sentido se volvió popular, tanto en el mundo académico como diplomático, lo que llamaremos de aquí en adelante, la "tesis del trampolín» que postula que una potencia emergente necesita, como precondición de su emergencia, ser potencia en su región respectiva. Así, por ejemplo, la brasileña Soares de Lima afirmó lo siguiente: «All regional powers that aspire to become global protagonists $[\ldots]$ must first be legitimated at the regional level since they do not possess enough material capacity or soft power to act autonomously in international politics» (citado en Malamud, 2011, p. 1). De la misma manera, Schoeman (2000) postuló que «the emerging power should be acceptable to its neighbors [...] A broader, or extraregional acceptance is perhaps a necessary condition, but not sufficient, even if supported and promoted by big powers» (p. 50).

Desde otra perspectiva, para Şaban Kardaş «regional powers are the nodes between the regional and global orders, as they work to balance the regional and global-level considerations in their foreign policy behavior» (2013, p. 645). En este sentido, según el investigador turco, se podría distinguir entre dos sentidos de interacción. Por un lado, la interacción outside-in, manifestada, por ejemplo, ante la penetración de una potencia extrarregional, donde la PR eventualmente puede aumentar su cuota de poder al entrar en una coalición con ella o ver una reducción de su influencia cuando dicha potencia se alía con rivales de la PR. Por otro lado, tanto las externalidades generadas por el grado de estabilidad regional (y el papel de la PR en ella), como el rol de «representante regional» asumido por la potencia regional, constituyen ejemplos de la interacción inside-out, desde el orden regional hacia afuera (p. 645). Además, agregamos que la conformación por parte de la PR de una alianza regional a fin de ejercer un mayor grado de autonomía frente a una potencia extrarregional, también puede generar un impacto en el sistema global.

\footnotetext{
${ }^{11}$ En su estudio sobre la política exterior de Brasil pos Guerra Fría, Burges estrenó el concepto de "hegemonía consensual» al considerar que a inicios del siglo XXI, el país no contó con los recursos suficientes — ni la voluntadpara asumir el costo de un proyecto de liderazgo explícito. Por ello, según el autor, "[t]he intent was not to seek Brazilian leadership of a South American region through imposition, but to instigate a mutually beneficial ordering that would quietly embed Brazilian interests, aspirations, and strategies in the region» (2009, p. 185).

12 Según Pedersen (2002), la hegemonía cooperativa «centers around the proposition that major states which are militarily weak or weakened may seek to maximize or stabilize their influence through non-coercitive means by pursuing a strategy of cooperative hegemony within a multilateral structure» (p. 696).
} 
Luego del reconocimiento de los problemas que puede traer el concepto de "potencia regional», llegamos a sus cuatro principales características — con sus respectivos indicadores-, tomando como referencia a los escritos de Nolte (2006 y 2010) ${ }^{13}$, Schirm $(2005)^{14}$, Flemes (2007) ${ }^{15}$ y Prys (2010). Primero, a nivel estructural, las PR cuentan con una preponderancia relativa de recursos, tanto de carácter material como ideacional. En otros términos, se trata de Estados «pivote» en su región respectiva. Mientras que el primero se manifiesta a través de indicadores tangibles, tales como el número de habitantes, el PBI, el gasto militar — todos como proporción del total regional- o índices compuestos como el $\mathrm{CINC}^{16}$, el segundo se percibe mediante muestras de prestigio o poder blando a nivel regional/internacional ${ }^{17}$. En segundo lugar, las PR no solamente se autoatribuyen el rol de potencia regional, sino que además contribuyen con la misma "construcción» o afirmación de su región. Tal como afirma Prys, dicho papel regional forma parte de las «concepciones del rol nacional» mencionadas por Holsti (entre ellas: el «excepcionalismo») y se manifiestan, aunque no siempre de modo explícito, mediante las declaraciones oficiales de las autoridades del Estado en cuestión (2010, pp. 12-13).

Tercero, las PR cuentan con una serie de instrumentos de política exterior, para movilizar sus recursos de poder. En este sentido, es muy común ver su participación en el establecimiento de una institución y/o agenda regional, la mediación en conflictos regionales, la representación de la región en espacios globales, el otorgamiento de bienes públicos (como preferencias comerciales, provisión de infraestructura o seguridad regional) o el ejercicio de una diplomacia pública proyectada hacia

\footnotetext{
${ }^{13}$ Nolte (2010) enumera once características de las PR, siendo las tres más importantes: la pretensión (u autopercepción) de ser líder en una región geográficamente, económicamente y políticamente delimitada; la posesión de recursos materiales e ideacionales para su proyección como potencia; y la influencia (a nivel de actividades y resultados) a nivel regional. De esta manera trata de conectar la dimensión estructural (principalmente, las capacidades de poder de las PR) con las estrategias de las PR, así como el resultado de las mismas.

${ }^{14}$ Schirm (2005) propone cinco características de una PR: pretensión de liderazgo; posesión de recursos materiales y organizacionales (power over resources), organización de actividades para honrar la pretensión de liderazgo y movilizar los recursos, reconocimiento del estatus de líder, y tener influencia en la región (power over outcomes).

${ }^{15}$ En su estudio sobre la política regional de Sudáfrica, Flemes menciona cuatro dimensiones de las PR: la pretensión de liderazgo; posesión de recursos materiales e ideacionales; proyección de instrumentos (materiales, institucionales y discursivos) de política exterior y; aceptación del liderazgo.

${ }^{16}$ El CINC (Composite Index of National Capability) constituye una medida de poder «duro» creada por el académico David Singer en el marco del proyecto "Correlates of War» (1963). Se determina como el promedio de seis porcentajes (sobre el total mundial) de las siguientes dimensiones: población, población urbana, producción de hierro y acero, consumo primario de energía, gasto militar o número de tropas. El último índice CINC se realizó en 2007 (http://www.nationalpower.info/cinc).

${ }^{17}$ Como otras dimensiones del poder, parte del poder blando de un país tiene que ser «activado» a fin de generar un impacto internacional, haciendo uso de ciertas estrategias que pertenecen a la diplomacia pública del Estado. Por ello mismo, consideramos que la proyección de poder blando mayormente se encuentra sujeta a la movilización de ciertos recursos económicos, como parte del poder duro.
} 
la región. Como tal, la elección de dichos instrumentos dependerá de la estrategia escogida por la potencia.

Finalmente, a nivel de recepción de la proyección de poder por parte de la PR (o en términos de Schirm, power over outcomes), se puede distinguir entre la percepción de los vecinos (y las resultantes políticas de seguimiento o balanceo) y la de terceros países, especialmente las potencias extrarregionales. La última característica se demuestra a través de discursos de los actores involucrados, encuestas de opinión pública o el grado de apoyo diplomático a la PR. Respecto a la aceptación por parte de los vecinos, cabe mencionar que esta no necesariamente implica aprobación. Así, incluso para el caso probablemente más "contundente» entre las PR, Sudáfrica, Flemes tuvo que constatar que: «Pretoria's regional leadership has been more acquiesced than welcomed, accepted or solicited» (2007, p. 44). Además, a lo largo de la presente investigación se enfatizará que, en buena medida, el reconocimiento regional de la PR dependerá de la percepción presente entre vecinos sobre si el país en cuestión cumple o no con las expectativas que generan los diversos roles asociados comúnmente con una potencia regional.

Como una síntesis de los tres conceptos brevemente elaborados hasta aquí, se podría postular que, pese a que no son sinónimos, por responder a tres diferentes lógicas, sí se interrelacionan estrechamente. En efecto, se considera que las potencias medias de segunda generación, que emergieron sobre todo a partir de la década pasada, se caracterizan por tener una proyección exterior más reformista y con vínculos más profundos con la región que las de primera generación, aunque comparten con estas últimas la proyección de poder blando (por ejemplo, en cuanto "país-modelo» o por el carisma de su líder). Además, si la emergencia de potencias puede basarse en su crecimiento económico y militar, se afirma a través de su proyección internacional más autónoma, principalmente mediante una política exterior más proactiva, asertiva y/o de carácter reformista, su impulso al multilateralismo y su liderazgo en coaliciones tanto regionales como extrarregionales, todo a fin de afirmarse como potencia emergente. Por otro lado, las potencias regionales, a raíz de su preponderancia material, su autopercepción como actor regional y el uso de ciertos instrumentos de política exterior para impulsar su papel como tal, pueden constituir un nexo entre el sistema global y los (sub)sistemas regionales. Finalmente, tanto las potencias emergentes como las regionales constituyen un estatus en la jerarquía internacional, resultado por cierto de su disponibilidad de recursos pero también del reconocimiento como tal por parte de los otros países, tanto en la región como afuera. Este reconocimiento a su vez se basa en el grado de legitimidad que obtiene, principalmente, a partir de un proceso permanente de negociación y su voluntad para contribuir con la provisión de bienes públicos (es decir, de su liderazgo global/regional). 


\section{Brasil y Turquía: las estrategias regionales de dos potencias medias emergentes}

Además de ser consideradas como potencias en cada una de sus regiones, Brasil y Turquía comparten otras características. En primer lugar, ambos países han sido aliados de Estados Unidos durante la mayor parte del siglo XX: Brasil desde los años diez con la «alianza no escrita» del canciller Barón de Rio Branco, mientras que Turquía desde el fin de la Segunda Guerra Mundial (y su incorporación a la OTAN en 1952).

En segundo lugar, tanto Brasil, siendo una nación lusófona y con una trayectoria sociohistórica muy diferente a sus vecinos; como Turquía, único país túrquico del Medio Oriente y además heredero del Imperio otomano, pueden ser vistos como outsiders en sus regiones. Precisamente estas diferencias con los vecinos explicaron parte de su histórica «indiferencia» y escasa identificación con la región. Sin embargo, a partir de la década de 1980, en un contexto interno de democratización, se iniciaría una notable profundización de las relaciones con el vecindario. Así, Brasil empezó con su histórico acercamiento hacia Buenos Aires, pieza clave para el incipiente regionalismo en el Cono Sur (y luego, Sudamérica). También Turquía, impulsado por el premier Turgut Özal (1983-1989) inició una diversificación de sus relaciones internacionales bajo el paradigma de bridge country, con el cual, por primera vez, se acercaría políticamente a los vecinos (y países musulmanes alrededor del mundo). No obstante, tanto la inestabilidad económica — Brasil entró en recesión en 1998, Turquía un año después- como la gran influencia del hegemón norteamericano en ambas regiones durante el «momento unipolar» socavaron dicha estrategia.

Tercero, durante la década pasada, ayudados por una sólida recuperación económica, la consolidación del régimen democrático, un liderazgo transformacional (ejercido por Ignacio Lula da Silva y Tayyip Erdoğan, respectivamente), una política exterior más proactiva y acompañada por un marcado deterioro de la imagen de EE.UU., ambos países empezaron a ser presentados como "modelos» en su región y reconocidos mundialmente como "potencias regionales». Por último, tanto Brasil como Turquía perdieron cierto protagonismo en sus regiones en el último lustro, como lo apreciaremos a continuación.

En cuanto a las diferencias, se pueden mencionar tres principales. Primero, respecto a su inserción geográfica, el Medio Oriente y Norte de África (MONA) y Sudamérica, obviamente, constituyen regiones muy diferentes entre sí. Si la primera sigue siendo uno de los ejemplos más dramáticos de una "formación conflictiva» (en términos de Buzan y Waever, 2004), básicamente por la histórica injerencia de las potencias occidentales y la tendencia de securitización de sus clivajes religiosos y étnicos, Sudamérica ha constituido un «régimen de seguridad» desde la década de 1980, aparentemente encaminado a convertirse en una "comunidad de seguridad» a mediano plazo. Vinculado a 
ello, Brasil se considera como un Estado geopolíticamente satisfecho con un proyecto nacionalista exclusivamente «de fines» (en función del desarrollo económico), mientras que Turquía sigue enfrentando relaciones complicadas con varios de sus vecinos (sobre todo Armenia y Siria) y su discurso es más propio de un nacionalismo étnico. Además, la estructura de ambas regiones es diferente: si Sudamérica se caracteriza por tener una estructura de poder prácticamente unipolar ante la preponderancia de Brasil frente a sus vecinos, en el MONA coexisten varias potencias regionales: más allá de Turquía encontramos a Irán, Arabia Saudita, Egipto, los Emiratos Árabes Unidos e Israel, que lo convierten en una configuración multipolar. Desde esta perspectiva, aparentemente se confirma la tesis, empíricamente comprobada por Lemke, que postula que mientras mayor sea la cuota de poder del país más importante en una región, más aumenta la estabilidad regional y mayor el número de organizaciones regionales (Nolte, 2010).

Finalmente, también el alcance de las proyecciones regionales de Brasil y Turquía difiere sustancialmente. El primero apostó, desde la década de 1990, por una identidad sudamericana de su política exterior, por encima del histórico término latinoamericano. Mientras tanto, Turquía se considera como una potencia «interregional». Por ello, el país está tratando de insertarse en varias regiones a la vez: más allá de sus históricos intentos de ingresar a la Unión Europea, impulsó su liderazgo en regiones como el propio MONA, pero también la Cuenca del Mar Negro, los Balcanes, Asia Central, así como «AfroEurasia» y el mundo musulmán, el último en cuanto espacio cultural. Reconociendo dicha proyección interregional de Turquía en las últimas décadas, la presente investigación considerará dos regiones: el MONA (con su configuración multipolar) y los Balcanes (históricamente en disputa entre potencias centroeuropeas, Rusia y Turquía).

Cabe mencionar que la estrategia regional de ambos países funciona paralelamente a otros mecanismos dirigidos a la emergencia global, tales como: la búsqueda de un mayor protagonismo en espacios multilaterales, la cooperación Sur-Sur, un discurso basado en el rol de «país-puente» (entre el Norte y el Sur para ambos, además del «puente cultural» en el caso turco), la mediación en conflictos y muestras de autonomía frente al hegemón en declive, Estados Unidos.

\subsection{La dimensión estructural: los recursos de poder}

A fin de determinar la preponderancia relativa de nuestros dos casos de PR en sus regiones respectivas (Sudamérica para Brasil y el $\mathrm{MONA}^{18}$ y los Balcanes ${ }^{19}$ para

\footnotetext{
${ }^{18}$ El MONA (20 Estados): Arabia Saudita, Argelia, Bahréin, Catar, EE.AA.UU, Egipto, Irak, Irán, Israel, Jordania, Kuwait, Líbano, Libia, Marruecos, Omán, Palestina, Siria, Túnez, Turquía y Yemen.

${ }^{19}$ Los Balcanes (10 Estados): Albania, Bosnia y Herzegovina, Bulgaria, Croacia, Grecia, Macedonia del Norte, Montenegro, Rumania, Serbia y Turquía. Pese a que Ankara se considera como un país balcánico, tan solo la región de Tracia pertenece geográficamente a dicha subregión europea. Además, cuatro Estados balcánicos ya pertenecen a
} 
Turquía), usaremos cinco indicadores: sus porcentajes de la población (CIA, 2018 ${ }^{20}$ ), el PBI (FMI, 2018 ${ }^{21}$ ) y del gasto militar regional (SIPRI, 2018 ${ }^{22}$ ), el CINC (National Power, $\left.2007^{23}\right)$, así como su posición en el ranking de los principales socios comerciales de los vecinos (FMI, 2018 24). Luego, nos detendremos en la dinámica, para el período 2000-2015, del comercio regional así como las inversiones en la región para los dos países (MIT, 2018 ${ }^{25}$ ). Finalmente, trataremos de realizar una estimación del poder blando tanto de Brasil como de Turquía.

Con más de 207 millones de habitantes —el 49\% de la población regional—; el $52 \%$ del PBI sudamericano y el $40 \%$ del gasto militar regional, Brasil claramente domina en su región. Esto también se refleja en el CINC donde Brasil llega al 50\% de Sudamérica, lejos de Colombia (12\%), Argentina (10\%) y Venezuela (9\%). Además, Brasil constituye el primer destino para las exportaciones de Argentina, Bolivia y Paraguay, el segundo para Uruguay, el sexto para Chile y Colombia y el octavo para el Perú. Respecto a las importaciones, Brasil representa el principal origen de estas para Argentina, el segundo para Bolivia, Paraguay y Uruguay, el tercero para Chile y el Perú, y el cuarto para Colombia (MIT, 2018).

En cambio, Turquía constituye la primera economía del MONA con el 21\% del PBI regional, antes de Arabia Saudita (17\%) e Irán (11\%) y, de lejos, la primera en los Balcanes (con el 58\%). A nivel demográfico, con casi 81 millones habitantes, Turquía cuenta con la tercera población del MONA, luego de Egipto (97 millones) e Irán (82 millones), mientras que domina en los Balcanes con el $57 \%$ del total regional. Respecto al gasto militar, Turquía representa apenas un $8 \%$ del total regional en el MONA, luego de Arabia Saudita (35\%), los Emiratos Árabes Unidos (12\%) e Israel (10\%) y, nuevamente, predomina con un 59\% en los Balcanes. Por otro lado, a nivel del CINC encontramos un empate entre Irán y Turquía (con 15\% cada uno), seguido por Arabia Saudita con 12\% del CINC del MONA, y un 56\% del índice para Turquía en los Balcanes. Finalmente, en el MONA Turquía constituye el primer origen de las importaciones para Irak, el tercero para Libia, el cuarto para Irán y el quinto para Túnez; solo figura en el top 5 de principales destinos para las exportaciones de Irán $\left(4^{\circ}\right)$. Mientras, en los Balcanes figura como segunda fuente

\footnotetext{
la Unión Europea y cinco son candidatos oficiales para ingresar al bloque. Como tal, es evidente que Turquía solo juega un papel secundario en la mayor parte de la región, por debajo de la UE y uno de sus Estados-miembro en particular; Alemania.

${ }^{20}$ Véase: https://www.cia.gov/library/publications/the-world-factbook

21 Véase: http://www.imf.org/external/pubs/ft/weo/2018/01/weodata/index.aspx

${ }^{22}$ Véase: https://www.sipri.org/databases/milex

${ }^{23}$ Véase: http://www.nationalpower.info/cinc/

${ }^{24}$ Véase: http://data.imf.org/?sk=9D6028D4-F14A-464C-A2F2-59B2CD424B85\&sId=1514498277103

25 Véase: https://atlas.media.mit.edu/en
} 
de importaciones para Albania y la quinta para Bulgaria; es el tercer destino para las exportaciones de Grecia y el cuarto para Bulgaria.

Más allá de los recursos (estáticos) de poder duro, cabe mencionar la creciente regionalización tanto de las exportaciones como de las inversiones extranjeras directas de Brasil y Turquía en las últimas décadas. Si en el caso brasileño, la regionalización de algunas de sus "multilatinas" ya empezó en la década de 1970, con un renovado impulso a partir de la primera década del siglo XXI, sus exportaciones intrarregionales recién empezaron a aumentar, en términos relativos, a lo largo de la década de 1990. Así, en el año 1990, apenas representó el 7,84\% del total para pasar a 17,10\% en 1995 y 18,57\% en 2000 (MIT, 2018). En este sentido, la conformación del Mercosur claramente contribuyó con la regionalización del comercio brasileño. A lo largo de los últimos veinte años, la proporción regional de las exportaciones de Brasil se ha mantenido estable con tasas entre el 15 y 20 por ciento (MIT, 2018).

Exportaciones regionales de Brasil y Turquía (1990-2016)

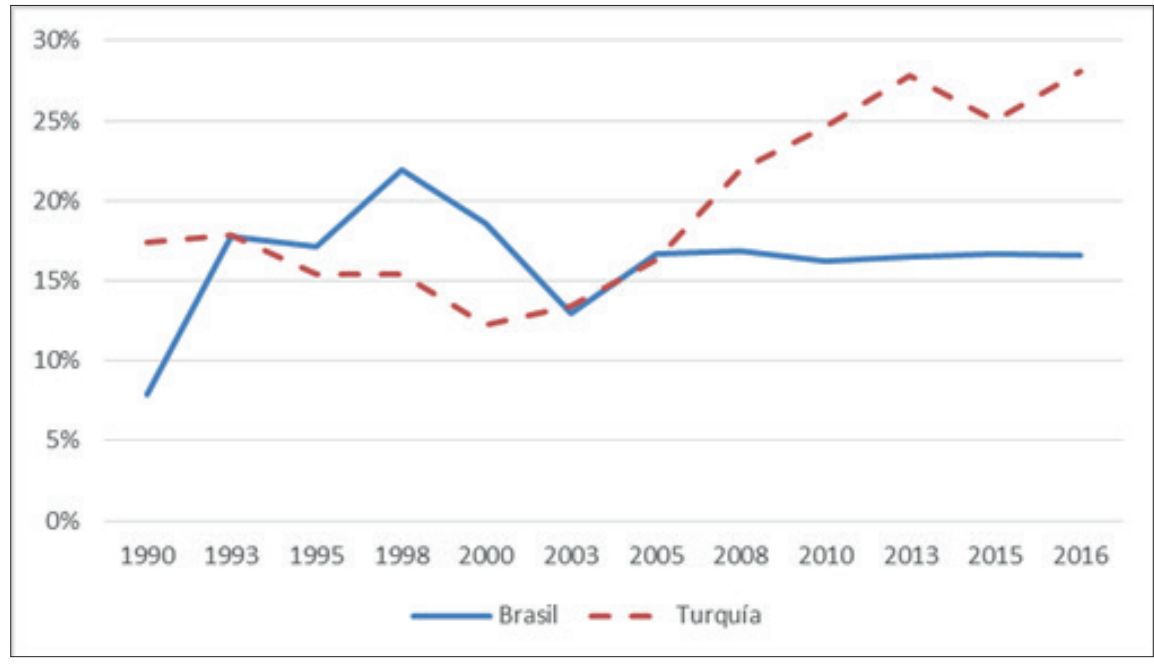

Fuente: MIT, 2018. Elaboración propia.

En cuanto a Turquía, mientras que la clase empresarial tradicional (mayormente proveniente de Estambul y Esmirna) históricamente se fue proyectando hacia el mercado europeo, la internacionalización de los denominados «tigres anatolios» a partir de la década de 1990 se concentró en mercados menos desarrollados, como los del MONA, los Balcanes, Asia Central y el Cáucaso. A nivel de las exportaciones, si durante décadas entre un 15 y $20 \%$ se dirigió a las regiones aledañas de Turquía, esta tasa primero fue bajando en la década de 1990 hasta un mínimo en el año 2000 de 
$12,21 \%$ para luego pasar por un boom en el transcurso de la década pasada: $16,33 \%$ en $2005,24,65 \%$ en 2010 y $28,13 \%$ en 2016 (MIT, 2018).

Según Joseph Nye (2004), el poder blando de un país se basa principalmente en tres ejes: su cultura, los valores y la política exterior. Mientras que analizaremos la política exterior de ambas potencias en el acápite $\mathrm{c}$, aquí revisaremos brevemente las primeras dos dimensiones. A nivel del poder ideacional, Brasil claramente cuenta con varias fuentes de poder blando. Más allá de contar con la reputación de ser un país pacífico (por ello la expresión; un «dinosaurio vegetariano»), defensor del derecho internacional y el multilateralismo y equipado con una cancillería altamente profesional, en general cuenta con una imagen internacional positiva, basada en su cultura popular (por ejemplo, mediante la música y las telenovelas), el deporte, las playas, el carnaval y su perfil multicultural. Además, a partir de la llegada al poder del presidente Lula da Silva, la consolidación del régimen democrático, el crecimiento económico en combinación con los grandes avances sociales, así como el carisma y activismo del presidente también contribuyeron con el poder blando del país ${ }^{26}$. Desde inicios del presente siglo, Brasil además se perfila como una "superpotencia alimentaria», una "potencia verde» y un líder de la diplomacia de salud. Es así que en el año 2012 Brasil llegaría al puesto 17 en la encuesta sobre poder blando de la revista Monocle ${ }^{27}$, siendo así el primer país del Sur en el ranking. Así también New Persuaders, una publicación del Institute for Government, lo ubicó en el puesto 20 para el año 2010 y 17 en 2012. Finalmente, en el Reporte Soft Power 30, recién estrenado en 2015, Brasil ocupó el lugar 23 para luego bajar al 24 en 2016 y el 29 en $2017^{28}$. Entre los principales desafíos a nivel de proyección de su poder blando citamos a la corrupción sistémica, la gran ocurrencia de homicidios, la desigualdad, así como el relativamente bajo Índice de Desarrollo Humano ${ }^{29}$. Además, el tamaño enorme del país y su histórico acomodamiento con Estados Unidos han generado la recurrencia de sospechas entre los vecinos sobre un presunto imperialismo brasileño. Así también lo reconoce el diplomático Paulo de Almeida (2010):

Being the largest economy in the region, Brazil exerts a natural attraction on neighboring countries, but that has not been enough to overcome old suspicions about its quasi-imperialistic behavior in South America, or its supposed desire to impose its own domination over other countries, replicating, albeit in a less arrogant manner, American imperial tutelage. (p. 171)

\footnotetext{
${ }^{26}$ En este sentido, como referencia, citamos al Latinobarómetro de 2010 donde el presidente Lula da Silva terminó como el segundo líder extranjero con mayor aprobación de la región (con 67\%), luego de Barack Obama (73\%) y antes de Hugo Chávez (33\%).

27 Brasil bajaría al puesto 22 en 2014 y 2015 y el 25 en 2017.

${ }^{28}$ Recuperado de: https://softpower30.com/wp-content/uploads/2017/07/The-Soft-Power-30-Report-2017-Web-1.pdf

${ }^{29}$ Por ello también, en su región Brasil no era/es considerado como un «modelo», tal como lo demuestra, por ejemplo, la encuesta del Latinobarómetro en el año 2011, donde 26\% consideró a Estados Unidos como «modelo a seguir», 19\% a Espańa y tan solo $11 \%$ a Brasil, aunque el último como primer país de la región.
} 
Mientras tanto, el soft power de Turquía se basa(ba) en la visión, muy poderosa hasta inicios de la presente década, de ser un "país-modelo" al combinar — como pocos países - una visión moderada del Islam, un desarrollo socioeconómico relativamente alto y la democracia ${ }^{30}$. Además, el país se presenta como un "puente» entre Europa (Balcanes) y Asia (MONA), un hub a nivel energético y en la aviación ${ }^{31}$, una referencia en el turismo global ${ }^{32}$ y el centro del mundo túrquico que se extiende desde Tracia hasta la provincia china de Xinjiang. Por otro lado, varios productos culturales de origen turco, sobre todo música popular y telenovelas, no solo tienen una audiencia masiva en los países vecinos sino hasta a nivel mundial. Finalmente, como en el caso brasileño, Turquía supo acumular poder blando por el activismo y liderazgo de su premier - actualmente presidente- Tayyip Erdoğan (como veremos, hasta 2011, aproximadamente) ${ }^{33}$. Por lo tanto, Turquía entró a la lista de Monocle en el vigésimo lugar para el año 2012 — luego bajó a la posición 26 en 2014 y 25 en 2015- y la posición 20 en el estudio del Institute for Government para 2012. En el Reporte Soft Power 30, el país ocupó el lugar 28 en 2015 y 30 en 2017. Entre los principales desafíos para su perfil de soft power, mencionamos su legado imperial ( $\mathrm{y}$ el actual «neootomanismo»), la presunta búsqueda actual de un nuevo imperio, la cuestión kurda irresuelta, el impacto interno de la gran inestabilidad de su entorno geográfico, así como el creciente autoritarismo y perfil sectario del gobierno turco a partir de 2011.

\subsection{La autopercepción y visión sobre el rol regional}

Como una segunda dimensión de su carácter como potencia regional, evaluaremos la autopercepción que prevalece en Brasil y Turquía, respectivamente, sobre su papel en la política internacional y regional.

\footnotetext{
${ }^{30}$ En una encuesta realizada en el año 2009 por TESEV en siete países árabes, un 61\% (incluso 73\% en Palestina) de los entrevistados consideró a Turquía como un «modelo», mientras que un $28 \%$ no lo vio así. En la misma encuesta, un $63 \%$ consideró al país como un «ejemplo exitoso de coherencia entre el Islam y la democracia». En otro estudio, también de TESEV, de 2011, un 78\% tuvo una imagen positiva de Turquía, alcanzando así el primer lugar antes de los Emiratos Árabes Unidos (70\%) y China (65\%). A su vez, según una encuesta del Gallup Balkan Monitor del ańo 2011, Turquía era considerada como un país amigable por un $93 \%$ de entrevistados en Kosovo (desde $62 \%$ en 2006), $71 \%$ en Macedonia del Norte (frente a 72\%), 69\% en Albania (contra 57\%), 52\% en Bosnia Herzegovina (contra 34\%), 34\% en Croacia, 31\% en Montenegro y 18\% en Serbia, reafirmando además la importancia del elemento cultural-religioso en este asunto (Herzog, 2014, p. 55).

31 Turkish Airlines es la única aerolínea del mundo en ofrecer destinos en más de cien países del mundo.

32 Turquía recibió casi 40 millones de visitantes en 2015.

33 Citamos, como ejemplo, al Arab Public Opinion Poll (de Zogby International y la Universidad de Maryland) de 2010, donde Tayyip Erdoğan figuró como el líder mundial más admirado con 22\%, seguido por Mahmoud Ahmadinajad (14\%) y Hugo Chávez (14\%). Barack Obama alcanzó un magro 2\% véase: https://www.politico.com/ pdf/PPM170_2010arabpublicopinion_power_point.pdf.
} 
Tal como se mencionó líneas arriba, durante gran parte de su historia, Brasil apenas se identificó con su región: si recién con la proclamación de la República en 1889, el país empezó a tildarse como «americano", no fue antes de la década de 1960 que surgieron los primeros gestos y discursos a favor de un mayor acercamiento hacia "América Latina» (por ejemplo, mediante su ingreso a la ALALC). No obstante, esta escasa identificación regional no excluyó la posibilidad de considerar, ya desde inicios del siglo XX, a la subregión sudamericana (con excepción de sus Estados caribeños) como una zona de influencia. Así, por ejemplo, durante el período militar (19641985), el concepto geopolítico de «los tres círculos concéntricos» era altamente influyente. Según esta visión, Estados Unidos figuró como potencia protagonista tanto a nivel global como hemisférica, mientras que Brasil debía ser el «árbitro dominante» en el círculo regional (Burges, 2009). Este rol de aliado e interlocutor de Washington y su gran crecimiento económico, además constituirían la base de la proyección brasileña en cuanto potencia emergente en la década de 1970, pero sin el afán de querer asumir el papel de coordinador o, menos aún, de representante de la región (Spektor, 2010). Entre los principales factores que explican esta «distancia cordial» encontramos las diferencias culturales e históricas con los vecinos; el tamaño del país (y, asociado a ello, el carácter ensimismado de la sociedad y la clase política brasileńa); las sospechas entre los vecinos sobre un supuesto imperialismo brasileño (unilateral o en alianza con Estados Unidos) y, durante mucho tiempo, la escasa interdependencia económica con la región. Además, pese a que desde 1910 Brasil mantuviera una política de "cordialidad oficial» con el mayor de sus vecinos, Argentina, hasta fines de la década de 1970 ambos países se consideraron como rivales.

Recién con la llegada al poder del último general de la junta militar en Brasil, João Figueiredo (1979-1985), se observaría un mejoramiento de las relaciones con Argentina y los demás vecinos del Cono Sur. Si esta reconciliación histórica fue, sobre todo, el fruto de la democratización en ambos países, también respondió a la creciente interdependencia económica, en marcha desde mediados de los setenta. Por otro lado, ante la creciente globalización del sistema capitalista y la narrativa de una regionalización de sus bases de producción, Brasil y Argentina tomaron la iniciativa de constituir el Mercosur, como fundamento de una incipiente integración, principalmente comercial, entre los países latinoamericanos. Desde sus inicios, el Mercosur además formó parte del esfuerzo brasileño para liberalizar su comercio e insertarse mejor en la economía mundial (Vigevani y Favaron, 2008, pp. 9-10).

Si la década de 1980 significó el gran avance en las relaciones de Brasil con los vecinos del Cono Sur y, al menos a nivel discursivo, su apoyo a favor de la solidaridad latinoamericana, a partir de la de 1990 empezaría a surgir un discurso innovador: el de la sulamericanidade. Fue durante una Cumbre del Grupo de Rio en el año 1993 
que el entonces canciller $-\mathrm{y}$ posteriormente: presidente- brasileño, Fernando Henrique Cardoso propusiera, por primera vez, el establecimiento de un organismo sudamericano: la Asociación para el Libre Comercio Sudamericano (ALCSA). Como una manera de justificar dicho cambio paradigmático, el ex canciller brasileño Celso Lafer (2002) comentaría lo siguiente, pocos años después:

América del Sur constituye una unidad física contigua, que favorece las oportunidades de cooperación económica. [...] A eso hay que añadir que, en el cambio del siglo, la economía de la geografía recomienda un nuevo enfoque para el concepto de América Latina. De hecho, hay que tener en cuenta que México, desde su participación en el NAFTA, y América Central y el Caribe, en virtud de la acción centrípeta de la economía norteamericana, vieron aumentar su grado de interdependencia con el Norte de manera aún más significativa en los últimos años. Por este motivo, el futuro de esta parte de América Latina está cada vez más vinculado con lo que ocurre en Estados Unidos. América del Sur, en contraste tiene relaciones regionales e internacionales más diversificadas, tanto en el plano económico como en el político. Este es un dato de la realidad contemporánea que le otorga una especificidad propia en el contexto de América Latina, del cual cabe extraer las consecuencias apropiadas en materia de política exterior (pp. 67-68).

Es decir, Brasil impulsaría a Sudamérica como nueva realidad económica y geopolítica, tomando en cuenta las ventajas que esto le podría traer para su inserción global, al excluir a su único «competidor» de América Latina, México, y al buscar mayor autonomía frente al hegemón Estados Unidos. Si la propuesta de ALCSA no recibió mayor apoyo entre los vecinos, sí hubo consenso regional, primero, para participar en una primera Cumbre presidencial exclusivamente sudamericana - lo que resultó en la creación de la Iniciativa para la Integración de la Infraestructura Regional Suramericana (IIRSA) - y luego, en 2004, para establecer la Comunidad Suramericana de Naciones (devenida en Unasur en 2008), un organismo con el mismo alcance geográfico pero un enfoque cada vez más político, en detrimento de la agenda comercial pendiente.

Con la llegada al poder de Lula da Silva, el regionalismo sudamericano empezó a convertirse en una prioridad para la política exterior brasileña. Así, durante su discurso de reinauguración, el entonces presidente afirmó lo siguiente:

A grande prioridade da política externa durante o meu Governo será a construção de uma América do Sul politicamente estável, próspera e unida, com base em ideais democráticos e de justiça social. [...] O Mercosul, assim como a integração da América do Sul em seu conjunto, é sobretudo um projeto político. Mas esse projeto repousa em alicerces econômico-comerciais que precisam ser urgentemente reparados e reforçados. [...] Apoiaremos os arranjos institucionais necessários, para que possa florescer uma verdadeira identidade do Mercosul e da América do Sul. Vários dos nossos vizinhos vivem hoje situaçōes difíceis. Contribuiremos, desde que chamados e 
na medida de nossas possibilidades, para encontrar soluçóes pacíficas para tais crises, com base no diálogo, nos preceitos democráticos e nas normas constitucionais de cada país. (Ministerio de Relaciones Exteriores de Brasil, 2007, p. 39)

No obstante, salvo en las declaraciones de algunos funcionarios del Itamaraty de menor rango, ni el presidente Lula ni su canciller Celso Amorim hablaron abiertamente en términos de un «liderazgo» regional. Por ejemplo, días después de su reelección, Lula da Silva declaró lo siguiente: «Hemos eliminado de nuestro diccionario cualquier atisbo de hegemonía. Brasil no quiere liderar nada, sino ser socio de todos los países y trabajar en armonía para que la gente pueda vea crecer nuestro continente» (Marirrodriga, 3 de noviembre de 2006). Dicha cautela claramente resultó de la escasa disposición del gobierno — - y, aún más de la sociedad brasileña - para invertir recursos en la provisión de bienes públicos regionales (véase más adelante). Por otro lado, el entonces canciller Amorim jamás ocultaría el claro vínculo entre el proyecto regional y las ambiciones globales de Brasilia:

South American integration is Brazilian foreign policy's top priority. Brazil recognizes that she is stronger and more influential in global affairs by working closely with her neighbors and by helping promote peace and prosperity in her region. In spite of her continental dimensions and vast natural resources, Brazil made a resolute choice to work for regional (i.e. South American) integration. (Amorim, 2007, p. 227)

En cuanto a Turquía, el país mostró dos grandes etapas en su política exterior hasta la década de 1980. La primera, desde la independencia en 1923 hasta 1945, se caracterizó por la estricta neutralidad, la no intervención (contrariamente al pan-islamismo y el turanismo) y la búsqueda de seguridad en cuanto nuevo Estado-nación ${ }^{34}$. Al mismo tiempo, los líderes del nuevo país eran conscientes de las ventajas que podían traer la ubicación estratégica y el «ingreso» de Turquía a Europa para su proyección regional. El propio fundador Kemal Atatürk (Altunişik, 2014) manifestó en 1927:

[...] that a Westernized, modern Turkey that had joined the community of civilization would have higher stature in the Muslim world as the messenger/ambassador of the West and Western values, than would a theocratic state. (p. 28)

A su vez, la segunda etapa refiere a la inserción de Turquía al campo occidental desde el inicio de la Guerra Fría. Así, luego de figurar como un país clave en la Doctrina Truman, estrenada en el año 1947, ingresó a la OTAN (1952), fue un país-fundador del Consejo de Europa (1949) y la OCDE (1961) y formuló un primer pedido de ingreso a la entonces Comunidad Europea en 1963. Al mismo tiempo, durante décadas Ankara mantuvo relaciones tensas no solamente con la Unión Soviética

\footnotetext{
${ }^{34}$ En este sentido, se suele referir al «Síndrome de Sèvres», el profundo temor que existió durante décadas en Turquía de quedar "partido" entre las potencias, tal como había sucedido con el Imperio otomano, luego del Tratado de Sèvres (1920).
} 
(y sus aliados), sino también con su histórico rival Grecia y los tres vecinos en el MONA (Siria, Irak y, luego de la Revolución islámica del 1979, Irán). Por estas razones, durante gran parte de la Guerra Fría, Turquía no solo era un "país-frontera" (border o flank country) del Occidente - luego de Noruega, fue el único miembro de la OTAN en compartir una frontera directa con la URSS — ${ }^{35}$, sino que también actuó como un promotor del statu-quo internacional y regional:

From the establishment of the Republic of Turkey in 1923 to the late 1980's, Turkey's foreign policy evolved around two main axes: the maintenance of the nation's Independence and the achievement of security in the face of Soviet ideological and territorial expansionism, and the preservation of the country's modernist, secularist, national regime. (Oskececi-Taner, 2005, p. 259)

Si la estructura bipolar propia de la Guerra Fría y las diferencias culturales eran principales causantes de la securitización de relaciones entre Turquía y sus vecinos, también contribuyó el protagonismo de las Fuerzas Armadas en su calidad de guardián del legado kemalista y co-arquitecto de la agenda de política exterior, tal como apunta Altunışık (2014):

[...] the Turkish military, which started to become a significant actor after the first military coup in 1960, adopted a defensive geopolitical discourse, arguing that "Turkey was located in a dangerous region and needed a strong army». This perception not only securitized foreign policy issues, thus allowing the military's influence in foreign affairs, it was also used as a justification for limiting democracy. (p. 31)

Con la llegada al poder de Turgut Özal (premier entre 1983 y 1989 y presidente de 1989 a 1993) se distinguiría un cambio en la proyección internacional de Turquía. Siendo un economista, Özal no solo fue el artífice de la liberalización de la economía turca llevada a cabo durante la década de 1980, sino que además consideró a la política exterior como un instrumento para alcanzar otros objetivos nacionales (Yilmaz, 2014).

Por un lado, la mejora de las relaciones con los vecinos podía fomentar la democratización de la política turca —al reducir el rol protagónico de los militares-. Por otro lado, una diversificación de las relaciones internacionales contraería grandes ventajas para la industria nacional, sobre todo para los denominados "Tigres de Anatolia», dirigidos por una nueva generación de empresarios provenientes del interior del país y con una identidad más religiosa que la burguesía tradicional de la costa (un perfil que compartió Özal al ser un creyente practicante). Si bien el concepto de "paíspuente» - es decir: una Turquía conectada a la vez con el Oeste y con el Medio

\footnotetext{
35 Según Buzan y Waever (2004) Turquía fue un ejemplo de un insulator state al no pertenecer a ningún complejo regional de seguridad: ni a Europa, ni tampoco al Medio Oriente.
} 
Oriente—, empezó a sonar desde la década pasada, fue con Özal que se convirtió en un verdadero paradigma de política exterior. En palabras del entonces premier:

We regard our existing ties with the Western World and close association with the Middle East and Islamic World as complementary elements of our foreign policy. Turkey's geographical location forming a natural bridge between the East and the West on the one hand and its common historical and cultural legacy on the other, require Turkey to attach importance to the Islamic World. (Yilmaz, 2014, p. 109)

Entre las iniciativas más importantes que acompañaron a esta política regional, mencionamos la creación de la Iniciativa de Asia Central (1991) y la Organización para la Cooperación Económica del Mar Negro (BSEC, por sus siglas en inglés, fundado en 1992), ambos en búsqueda de una mayor conexión con países del espacio ex soviético, así como las acciones de mediación por parte de Turquía durante los conflictos de Nagorno Karabaj y en Bosnia Herzegovina.

Si Özal realizó los primeros pasos en la apertura hacia la vecindad, fue con la llegada al poder en 2003 de Tayyip Erdoğan que Turquía empezó a apostar por una estrategia explícitamente regional. Un hito en este proceso fue la publicación, dos años antes, del libro Profundidad estratégica del entonces académico Ahmet Davutoğlu ${ }^{36}$, escrito a fin de revisar el rol regional y global de su país. Según el autor, Turquía cuenta con una "profundidad» tanto histórica como geográfica en su vecindario. A nivel histórico, de la misma manera como Francia, Rusia o China, durante siglos Turquía no solo se había ubicado en el «epicentro de la historia mundial», sino, además, a partir del legado otomano, mantenido la responsabilidad para desarrollar relaciones multidimensionales con los vecinos (Fisher, 2012, pp. 63-64). Además, el libro postula que fue el propio colapso del Imperio otomano y la posterior emergencia de EstadosNación árabes artificiales que habían originado la desintegración política y económica del mundo islámico en su conjunto. Ahora, según Davutoğlu, luego de dicho «paréntesis» en el orden regional del Medio Oriente, Turquía tuvo que rehabilitar la era otomana, devenida un tabú en la política turca desde la proclamación de la república, y «reintegrarse» con sus vecinos (Özel y Özkan, 2015).

A su vez, la profundidad geográfica refiere al aprovechamiento de la posición altamente estratégica de Turquía en el cruce de varias cuencas terrestres (los Balcanes, el Cáucaso y el MONA) y marítimas (el Mar Negro y Caspio, el Mediterráneo y el golfo Pérsico), cruciales en la geopolítica global. De esta profundidad estratégica

\footnotetext{
${ }^{36}$ Luego de una carrera como profesor de Ciencia Política y Relaciones Internacionales, Ahmet Davutoğlu se convirtió en 2003 en asesor presidencial para política exterior del entonces premier Tayyip Erdoğan hasta ocupar el cargo de ministro de Relaciones Exteriores de 2009 a 2014. En este ańo, ya como líder del Partido (oficialista) de Justicia y Desarrollo (AKP) fue premier hasta mayo de 2016, momento en el cual, aparentemente, se distanció del ahora presidente Erdoğan.
} 
resulta la proyección a futuro de Turquía como un "país central» en la política regional y mundial, y no meramente un "país-puente» entre regiones, considerado como un rol internacional demasiado pasivo. Finalmente, Davutoğlu formuló los cinco principios propios del nuevo «enfoque visionario» en la política exterior turca: 1) el equilibrio entre libertad y seguridad (sobre todo por el carácter multinacional de la cuestión kurda); 2) la búsqueda de "cero problemas con los vecinos»; 3) el ejercicio de una diplomacia de paz preventiva y proactiva, basada en el diálogo estratégico, la integración económica y la coexistencia cultural; 4) adherencia a una política exterior multidimensional, enfocada en la cooperación con todos los protagonistas en la política global; y, 5) una diplomacia «rítmica» sentada en el multilateralismo, la flexibilidad y el empoderamiento en política exterior de varios nuevos actores (también no estatales).

No constituye una exageración afirmar que desde la llegada al poder del AKP de Erdoğan en 2003 y, aún más, de Davutoğlu como canciller en 2009, la visión descrita se convirtió en la guía de la política exterior de Turquía. O, como lo entendió Cagaptay (16 de agosto de 2013):

If Atatürk saw Turkey as the Argentina of the Middle East, a country physically in the region but mentally in Europe, the AKP envisioned Turkey as the Brazil of the Middle East, a rising economic power with a burning desire to shape regional events. (p. 1)

Varios factores provocaron esta revisión paradigmática de la proyección internacional de Turquía a inicios de la década pasada. Por un lado, a nivel doméstico, ciertamente contribuyó el gran crecimiento económico y, en paralelo, la rápida expansión del comercio e internacionalización de numerosas empresas turcas; el creciente perfil islámico del entonces premier; así como la de-securitización de la cuestión kurda. En cambio, a nivel internacional, coincidió con el deterioro de la imagen de Estados Unidos a partir de la intervención en Irak, una nueva escalada en el conflicto entre Israel y las autoridades palestinas y, al menos en su primera etapa, la Primavera Árabe, cuando en varios países del MONA se empezó a referir a Turquía como un modelo en la región. Sobre todo los dos primeros aspectos del contexto internacional facilitaron de cierta manera la búsqueda de Erdoğan de autonomía frente a Washington (y el Occidente, en general), el tono a veces abiertamente revisionista en espacios multilaterales y el distanciamiento manifiesto entre Ankara y Tel Aviv. En este sentido, la aprobación de la resolución parlamentaria en 2003 que imposibilitó el uso de bases turcas para tropas estadounidenses, la ruptura en 2009 de relaciones diplomáticas entre Turquía e Israel y el establecimiento en 2012 de una asociación estratégica entre Turquía y la Organización para la Cooperación de Shanghái, contra un fondo de apertura hacia los vecinos, constituyeron tres hitos históricos.

Contrariamente a Brasil, en Turquía no siempre hubo sintonía entre el premier y otros funcionarios clave del gobierno. Así, Tayyip Erdoğan manejó un discurso rela- 
tivamente cauteloso respecto al papel de Turquía en la región. Por ejemplo, postuló lo siguiente en 2014:

Turkey has no objective of becoming either a regional or global power. Turkey is being positioned in the region and in the international community for simply doing its part. That's how it should be. The opposite is defined as ambition and ambition is always dangerous. We therefore have no such ambition (Hürriyet, 8 de enero 2014).

Así también, durante su famosa gira por el mundo árabe, recién iniciada la Primavera Árabe, presentó a su país como un aliado, más que un líder: «Enough we say, the decision belongs to the people of the brotherly Egyptian and Tunisian nations... Turkey shares the grief of these nations as well as their hopes» (Walker, 25 de mayo de 2011).

Mientras tanto, en línea con el mensaje de su libro de 2001, Davutoğlu reconoció públicamente las ambiciones regiones y globales de su país. Describió, por ejemplo, al MONA (y el «espacio otomano») como un "binterland necesario» de Turquía y su "destino manifiesto», mientras que enfatizó la pertenencia regional del país (regional ownership). En este sentido, durante un discurso ante el Parlamento turco en 2011, afirmó lo siguiente: «We are trying to implement this 'strategic depth' in order to make Turkey a global actor $[\ldots]$ this is the essence of the foreign policy which we are attempting to put into practice every day». Meses después, ante el mismo pleno, incluso expresó las hoy famosas frases: "A new Middle East is about to be born. We will be owner, pioneer and the servant of this new Middle East» (Hürriyet, 27 de abril 2012). A su vez, defendió la así llamada Pax Otomanica durante una visita a Sarajevo en 2009:

Like in the 16th century, which saw the rise of the Ottoman Balkans as the center of world politics, we will make the Balkans, the Caucasus and the Middle East, together with Turkey, the center of world politics in the future. This is the objective of Turkish foreign policy, and we will achieve this. We will reintegrate the Balkan region, the Middle East and the Caucasus, based on the principle of regional and global peace, for the future, not only for all of us but for all of humanity. (ESI, 4 de diciembre de 2010)

Por otro lado, el ex canciller solía conectar su política exterior «neo-otomana» con la creciente regionalización de la economía turca:

We want our economic borders, frontiers bigger than our political frontiers. Of course, we respect the territories. And it is not an era of conquest. No. And we do not have any imperial objective. Neither in the Middle East nor in the Caucasus nor in the Balkans. But what we want is, we want free mobilizing of economic resources, especially free mobilization of our manpower, of our businessman, through the world. [...] When you look at Turkey, do not see only a country with 786.000 square kilometers. But you see a hinterland; you can see where you can reach from Istanbul. [...] So this policy of reintegration [...] with our neighbors will continue: Antep and Aleppo, Edirne and Plovdiv, Istanbul and Selanik, Izmir and Athens, Rize and Batumi will be reintegrated. (Davutoğlu, noviembre de 2011) 
Finalmente, cuando ya había empezado la Primavera Árabe, Davutoğlu pasó a describir el rol turco frente a los vecinos como un order instituting actor, tratando así a la región, más que como una «zona de interés», como una "zona de influencia» (Özel y Özkan, 2015). De la misma manera, el presidente Abdullah Gül (2007-2014) se refirió a Turquía como un regional power house, pese a que describió a su país como una «fuente de inspiración», más que un modelo para los vecinos (Parlar, 2016).

De esta manera, luego de varias décadas de «desinterés» hacia la región, tanto Brasil como Turquía empezaron a percibir a la región como una prioridad en su agenda de política exterior a partir de la década de 1980. Sin embargo, fue recién en la década pasada que los dos empezaron a postular una estrategia explícitamente regional: el paradigma de la sulamericanidade para Brasil y el de "profundidad estratégica" y "país central» en el caso turco. Todo ello en un contexto de expansión económica, acumulación de poder blando, líderes transformacionales y la narrativa sobre un imperial overstretch del hegemón. La principal diferencia entre ambos países queda en el grado de asertividad: mientras que Brasil siempre trató de evitar cualquier referencia al rol de líder regional, Turquía — sobre todo el arquitecto de la nueva política exterior turca - no tuvo las mismas reservas sobre el caso, al aludir al pasado otomano y usando un tono propio del excepcionalismo.

\subsection{Las estrategias y roles de política exterior}

En el presente acápite se realizará un breve repaso de las estrategias regionales implementadas por ambas potencias en los ámbitos de la diplomacia tradicional y la pública, así como de sus roles de «líder intelectual», mediador, paymaster y representante, respectivamente.

\subsubsection{Las estrategias de diplomacia tradicional y pública hacia la región}

Más allá del papel que tuvo Brasil en auspiciar la primera cumbre presidencial sudamericana (2000), también usó otros mecanismos (bilaterales) para acercarse hacia los vecinos. Así, a partir de la llegada al poder del presidente Lula da Silva, aumentó considerablemente el número de visitas oficiales del presidente y del canciller Celso Amorim a los países de la subregión. En el mismo periodo, Brasil además firmaría acuerdos de alianza estratégica con tres principales vecinos: Perú (2003), Argentina (2004) y Venezuela (2005), abarcando una serie de temas de cooperación (entre otros; energía, infraestructura, seguridad transfronteriza y salud). Por otro lado, como efecto de la democratización y la apertura del Itamaraty hacia otros actores no estatales, Brasilia promovería una "diplomacia social» en la región protagonizada por algunos movimientos sociales - al tomar como precedente al Foro Social de Sao Paulo- y el propio Partido de Trabajadores. Por cierto, esta tendencia originó el creciente perfil ideológico de la política exterior brasileña durante estos años, tal 
como se manifestó con las numerosas muestras de apoyo a candidatos progresistas durante las elecciones en países vecinos. Finalmente, mencionamos al creciente énfasis puesto por la cancillería brasileña, mayormente desde la década pasada, en su departamento cultural a través de la Red Brasil Cultural, a cargo de la promoción del uso de la lengua portuguesa, y en los Centros Culturales Brasileños.

En lo que concierne Turquía, en el marco de la política «cero problemas con los vecinos» se empezó a observar una dramática mejora de las relaciones bilaterales a partir de 2003. Al igual que con el caso brasileño, hubo un gran aumento en el número de visitas oficiales tanto del premier, como de otros funcionarios gubernamentales, fomentando así la confianza personal entre los mandatarios: solo Erdoğan realizó no menos de 55 visitas oficiales a los países del MONA durante su premierato (2003-2014) (Eksi, 2016). Además, a partir de 2005, Turquía tomó la iniciativa de convocar «Consejos de Cooperación Estratégica de Alto Nivel» —el equivalente de los gabinetes binacionales en América del Sur- con dieciséis países, sobre todo los vecinos del MONA y los Balcanes. De esta manera, Ankara logró acercarse a países como Siria, Armenia y Grecia, que pocos ańos antes eran vistos con hostilidad. Finalmente, en el ámbito de la diplomacia cultural, Turquía invirtió numerosos recursos en la creación en 2009 de una red de institutos llamada Yunus Emre, con presencia en los Balcanes (Sarajevo obtuvo la primera sede), seguido por países del MONA (siendo El Cairo el pionero en dicha política). Para 2015, Turquía ya contaba con 41 centros en 32 países de dicho Instituto (Eksi, 2016). De modo paralelo, desde el partido oficialista AKP se ha enfatizado mucho la identidad religiosa de Turquía. No obstante, tal como comenta Eksi: «Muslim identity was pragmatically used as a hegemonic instrument in order to open a space in the Middle East policy with a model country identity as soft power, to be accepted by Muslim communities, to win their sympathy and support» (Eksi, 2016, p. 69). Como tal, el gobierno de Erdoğan condujo una política de nation branding en el MONA, basada en la identidad y el modelo de su partido. Sin duda una de las principales ilustraciones de esta "diplomacia religiosa» consiste en el proyecto Hadiz, en manos del Directorio de Asuntos Religiosos (Diyanet), que se esfuerza a reinterpretar dicha fuente de la ley musulmana de acuerdo con la realidad del siglo XXI.

\subsubsection{Construcción de la región, agenda setting e institucionalización regional}

Tal como lo mencionamos anteriormente, Brasil fue el país que lideró la delimitación y construcción intelectual de la región «Sudamérica», en detrimento de otras (macro-) regiones ya existentes, como el Hemisferio Occidental, América Latina o el propio Cono Sur. Si la sulamericanidade ya estuvo presente en el vocabulario del Itamaraty en la época del Barón de Rio Branco (a inicios del siglo XX), fue recién a partir de 1993 que empezó a dominar con la propuesta del ALCSA y, aún más, a partir de la 
primera cumbre sudamericana de Brasilia en 2000. En dicho ańo, considerando la sensibilidad que generó entre los vecinos la propuesta brasileña de conformar una zona de libre comercio, el entonces presidente Cardoso avanzaría con propuestas de otra índole, siendo la más conocida la iniciativa IIRSA a fin de fomentar la integración física de la subregión, y la idea sobre la necesidad del diálogo político y la concertación entre los países sudamericanos. Luego, durante la Cumbre del Cusco en 2004, Brasil tomó la iniciativa para institucionalizar las cumbres sudamericanas mediante la Comunidad Sudamericana de Naciones (o CASA). Finalmente, en 2008, bajo influencia venezolana, la CASA se convertiría en Unasur, dentro del cual Brasil lideró la creación de varios consejos sectoriales, como el de Defensa y de Salud. Pese a este liderazgo intelectual, Brasil siempre se opuso a cualquier tipo de institucionalidad regional que podría afectar a su autonomía, una de las premisas históricas de la política exterior del país. Además, logró «neutralizar» propuestas abiertamente anti-hegemónicas provenientes de los países del ALBA.

En cambio, más allá de haber liderado el incipiente regionalismo en la Cuenca del Mar Negro de la década de 1990, durante toda la década pasada, Turquía no se encargó de construir o delimitar una nueva región. El país se presenta, a la vez, como «potencia interregional» (en regiones ya existentes), pivote en el espacio geopolítico de "Afro-Eurasia» y líder del mundo islámico. Más que referencias al legado otomano, tampoco hubo el intento de recrear dicho espacio histórico con iniciativas concretas a nivel multilateral. Por otro lado, Turquía sí firmó acuerdos de asociación con organismos regionales - mencionamos el mecanismo de diálogo estratégico con el Consejo de Cooperación en el Golfo (2005) y el «Foro de Cooperación TurcoÁrabe» con la Liga Árabe (2006) — y logró ocupar el cargo de secretario general de la Organización de Cooperación Islámica (2005), pero sin crear nuevas instituciones regionales. En otros términos, la normalización de relaciones y cooperación con los vecinos se implementó básicamente a nivel bilateral. De esta manera, Turquía firmó acuerdos de libre comercio con Siria (2004), Palestina (2005), Túnez (2005), Marruecos (2006), Albania (2006), Montenegro (2008) y Jordania (2011, suspendido en 2018). Así también suscribió acuerdos bilaterales de exención de visa con todos los países balcánicos, Catar, Irán, Israel, Jordania, Kuwait, Líbano, Marruecos, Siria y Túnez. Basándose en esta experiencia, recién en diciembre de 2010, Turquía concluiría un acuerdo para establecer, conjuntamente con Jordania, Líbano y Siria, el "Cuarteto del Levante», entendido como el primer paso hacia la formación de un mercado común, siguiendo claramente los pasos de la $\mathrm{UE}^{37}$, donde posteriormente podrían participar los países árabes del Golfo e Irán (Hürriyet, 3 de

\footnotetext{
${ }^{37}$ En una clara alusión a la Zona Schengen de la UE, funcionarios turcos describieron al Cuarteto en términos de la Zona Shamgen (de la palabra árabe al Sham o Levante).
} 
diciembre 2010). Sin embargo, el inicio del conflicto sirio, meses después, hizo que dicho acuerdo no prosperara.

\subsubsection{Diplomacia de mediación}

Ante la ausencia de conflictos interestatales de gran intensidad en Sudamérica durante las últimas décadas, Brasil no tuvo que recurrir a la mediación entre sus vecinos. La única excepción en este sentido ocurrió en 1995, cuando el país jugó un papel crucial en el proceso de paz entre Ecuador y el Perú ${ }^{38}$. A su vez, más allá de la intervención en Paraguay en 1996, a fin de evitar un golpe militar en dicho país, el «coloso sudamericano" se mostraría fiel ante el principio de no intervención y la no injerencia en asuntos internos de otros Estados. Así mismo, durante la década pasada, Brasil actuó como mediador en algunas de las crisis políticas de sus vecinos pero dando preferencia al enfoque multilateral, aparentemente, a fin de no despertar recelos o sospechas sobre un presunto imperialismo brasileño. Por ejemplo, durante el último año de la presidencia de Cardoso (2002), Brasil tomó la iniciativa de conformar el grupo de los "Amigos de Venezuela» para incentivar el dialogo entre el (primer) gobierno de Hugo Chávez y la oposición. Pese a que el canciller brasileño de Lula da Silva, Celso Amorim, enfatizaría la importancia de la não-indiferença, como supuesta alternativa de la não-intervenção ${ }^{39}$, en el fondo Brasil dio continuidad a dicha línea durante los años del PT. De esta manera, Brasil ayudó a mediar, junto con Argentina, durante la crisis boliviana entre 2003 y 2005 y ante la turbulencia política en Ecuador que llevó al derrocamiento del presidente Lucio Gutiérrez en 2005. Así también, fue bajo impulso de Unasur que se llegó a una salida de la crisis política boliviana de 2008 y de la crisis diplomática entre Colombia y Venezuela en 2010. Cabe mencionar que el ejército brasileño además lideró el componente militar de la Misión de Estabilización de la ONU en Haití (Minustah), compuesta principalmente por tropas sudamericanas. Sin embargo, Brasil no tuvo un papel importante en las negociaciones de paz en Colombia y tampoco logró mediar en los últimos años entre el oficialismo y la oposición en Venezuela.

En cambio, a partir de la década pasada, el rol de mediador se ha convertido en un elemento clave de la representación internacional de Turquía en la región y el mundo. Mientras que en algunos casos Ankara apoyó iniciativas multilaterales como el Pacto de Estabilidad de Europa Suroriental en los Balcanes o las misiones de la OSCE en el Cáucaso, Ankara pasó a presentarse como mediador de iniciativa propia. Así, medió

\footnotetext{
${ }^{38}$ Brasil no solo logró impulsar un alto al fuego y la firma de un acuerdo de paz en este conflicto, sino que además lideró la Misión de Observadores Militares Ecuador-Perú (Momep).

39 Así, por ejemplo, cuando Brasil empezó a liderar la misión Minustah en Haití declaró lo siguiente: «[...] nós não acreditamos em interferência externa em questóes internas, mas não procuramos refúgio na omissão e indiferença perante os problemas que afetam nossos vizinhos» (Spektor, 2010, p. 29).
} 
entre Israel y Siria a fin de llegar a un acuerdo de paz (2008), entre Hamas y Fatah en la Franja de Gaza (2011) y, además, participó en las negociaciones de paz en Afganistán, Myanmar y Somalia. Estableció varios «Procesos trilaterales» de reconciliación (Turquía-Bosnia-Croacia, Turquía-Bosnia-Serbia y Turquía-AfganistánPakistán) y lanzó las plataformas diplomáticas «Vecinos de Irak» y la de «Estabilidad y Cooperación en el Cáucaso». Turquía y Brasil también crearon un mecanismo de negociación, alterno al «P5+1», para superar la crisis nuclear iraní. Finalmente, para reforzar la idea de ser un "país-mediador» ante la comunidad internacional creó, junto con Finlandia, la plataforma "Amigos de Mediación», bajo el marco de las Naciones Unidas (Davutoğlu, 2013). No obstante, a raíz de su papel en el conflicto sirio desde 2011, la imagen de mediador (y, aún más, la de ser un soft power en el MONA) ha quedado altamente afectada en los últimos ańos. Como tal, queda por ver si la participación turca en el Proceso de Paz de Astana a fin de buscar un diálogo político en Siria pueda revertir esta percepción.

\subsubsection{El rol de paymaster: la provisión de bienes regionales}

Entre las principales muestras de «solidaridad» de Brasil hacia sus vecinos sudamericanos en el periodo 2000-2015, citamos a tres mecanismos. En primer lugar, en 2003 se lanzó el «Programa de Substitución Competitiva de Importaciones» (o PSCI) al argumentar que la sustitución de importaciones desde el resto del mundo, por productos provenientes de Sudamérica — de modo particular: los vecinos que no integraban el Mercosur - contribuiría al crecimiento económico y la profundización de la integración regional (Couto, 2007). Si bien dicho mecanismo contribuyó con el gran aumento de las exportaciones de algunos vecinos hacia el mercado brasileńo durante la década pasada, claramente no fue suficiente para revertir la balanza comercial deficitaria de los mismos.

Luego, en 2004, se creó el Fondo para la Convergencia Estructural del Mercosur (Focem) en un intento de combatir las asimetrías al interior del bloque. Inspirado en experiencias similares de la Unión Europea, el Fondo financió varios proyectos de desarrollo, sobre todo en los sectores de vivienda y transmisión eléctrica de los dos miembros menores del bloque, Paraguay y Uruguay. Sin embargo, luego de diez años, el Fondo solo había desembolsado la suma de, aproximadamente, mil millones de dólares, con un $70 \%$ de la suma proveniente de Brasil, un número relativamente bajo al considerar el tamaño de la economía brasileña (Focem, 2015). Finalmente, algunos países de la región, tales como Bolivia, Paraguay y el Perú, recibieron apoyo económico de forma bilateral o a través de la $\mathrm{ABC}$, la Agencia Brasileña de Cooperación. Esta cooperación técnica se implementó mayormente en sectores como educación, agricultura, salud y ciencia (Saraiva, 2012). 
Más allá de estas modestas contribuciones, los gobiernos del PT mantuvieron una política denominada "paciencia estratégica» hacia los vecinos, cuando algunos de ellos implementaron políticas abiertamente nefastas para los intereses de Brasilia. Esto ocurrió, por ejemplo, frente a la imposición unilateral por parte de Argentina de salvaguardias comerciales (2005); luego de la «nacionalización» en Bolivia de las instalaciones de hidrocarburos en manos de Petrobras (2006) o cuando Paraguay le exigió un pago adicional por la generación de energía eléctrica en la central de Itaipú (2009). Pese a lo anterior, a raíz de los grandes desafíos socioeconómicos internos y al escepticismo de la opinión pública brasileña hacia una política de generosidad frente a la región, Brasil no invirtió numerosos recursos en esta durante el periodo estudiado $^{40}$.

En el caso turco, el rol de paymaster quedó cubierto por la Agencia Turca de Cooperación y Coordinación (o TIKA), un mecanismo crítico en la creciente proyección del país como líder de la «diplomacia humanitaria» o rising donor (Eksi, 2016). Siguiendo la perspectiva de ser una potencia multiregional, para el ańo 2014 Ankara ya contó con más de cincuenta oficinas de coordinación de programas a nivel mundial, principalmente ubicadas en los Balcanes, el MONA, el Sahel y Asia Central, siendo los principales receptores de la AOD turca Palestina, Afganistán, Albania, Bosnia Herzegovina, Kirguistán y Somalia (TIKA, 2014 y OECD, 2017). Sumando la asistencia pública y privada, Turquía incluso llegó a convertirse en un líder mundial de ayuda humanitaria: según cifras de la OCDE de 2017, el país logró invertir el 0,95\% de su PBI en AOD (desde un 0,45\% en 2014), solo por debajo de los EE.AA.UU., y en un nivel similar a Suecia y Noruega ${ }^{41}$. Esto sí, según el Reporte de Asistencia Humanitaria Global del año 2017, casi el 99\% de los 6 mil millones de dólares respondió a programas de ayuda para los más de tres millones de refugiados sirios hospedados en territorio turco (Devinit, 2017).

\subsubsection{El rol de representante de la región}

Históricamente, la política exterior brasileña no se ha caracterizado por procurar posiciones de bloque con sus vecinos a nivel multilateral: si el país ha tratado de alcanzar el estatus de "gran potencia», siempre lo hizo partiendo de su propia capacidad y no en representación de la región. Sin embargo, el país sí ha buscado en algunas ocasiones el apoyo de los vecinos para causas individuales. Así sucedió como miembro de la Sociedad de Naciones (1919-1926), durante sus intervenciones en

\footnotetext{
40 De la misma manera, Brasil tampoco ha figurado como un paymaster en la gobernanza global. Así en el año 2011, las contribuciones brasileñas a la OMC, pese a ser una organización importante para la proyección externa de Brasilia, solo alcanzaron la mitad de aquellas de un país como Bélgica; y en el FMI llegaron a ser la mitad de aquellas aportadas por Italia (Gardini, 2016).

41 Véase: http://www.oecd.org/dac
} 
la Asamblea de las Naciones Unidas y en su calidad de defensor de los intereses económicos de los países en vía de desarrollo en el marco de las rondas del GATT —en cada uno de los cuales Brasil se presentó en términos de «representante de la región»—. Además, durante los años que figuró como miembro no permanente del Consejo de Seguridad, solo en raras ocasiones pasó a representar a sus vecinos. Así también ha ocurrido en el marco de la OMC, donde inicialmente Brasilia lideró al G23 (doce de sus miembros son de América Latina), para luego, durante la Sexta y Séptima Conferencia Ministerial en Hong Kong (2005) y Ginebra (2009), respectivamente, aceptar las propuestas de los países industrializados. Finalmente, con excepción de la organización de una cumbre en 2014 entre Unasur y BRICS, tampoco ha dado muestras de representación alguna en nuevos foros como este último o el G20.

En cuanto a Turquía, su rol como representante de sus vecinos ha sido algo más marcado. Así, sobre todo a partir de su segundo gobierno, Erdoğan empezó a convertirse en uno de los principales voceros en defender la causa palestina, siendo el momento más recordado cuando formuló duras críticas durante el Foro Económico Mundial de 2009 en contra de Shimon Peres. Además, Ankara ha actuado como portavoz de varios de sus vecinos en su candidatura para ingresar a la OTAN, empezando con sus cuatro principales socios en los Balcanes (Albania, Bosnia, Kosovo y Macedonia del Norte), así como Azerbaiyán y Georgia. Así también, Turquía se ha presentado como el representante del mundo islámico en la misma OTAN, la Unión Europea y espacios multilaterales como el G20 o las Naciones Unidas (por ejemplo, mediante la "Alianza de las Civilizaciones», cuyo co-auspiciador era el propio Erdoğan). En estos espacios, finalmente, Ankara realizó llamadas a favor de una reforma en la arquitectura de las instituciones multilaterales, de mayor atención a favor de la miseria en el Sur o de una perspectiva más multicultural en la gestión de los desafíos mundiales ${ }^{42}$. Como ilustración de dicho estilo reformista, incluimos la siguiente cita de Erdoğan durante una entrevista en 2011: «The world is changing to a system where the will of the people will rule. Why should the Europeans and Americans be the only ones that live with dignity? Aren't Egyptians and Somalians also entitled to a life of dignity» (The Guardian, 13 de setiembre de 2011).

\footnotetext{
${ }^{42}$ En este sentido, Davutoğlu durante un discurso a fines de 2011 aseveró «We think that in UN there should be a much more participatory political order, much more justice oriented and economic order and a much more inclusive cultural order» (Ministerio de Relaciones Exteriores de la República de Turquía [MFA], 2011).
} 


\subsection{La recepción por parte de la región y las grandes potencias}

Como última dimensión en su calidad de potencia regional, trataremos la recepción por parte de los vecinos y las grandes potencias de la estrategia regional de Brasilia y Ankara.

\subsubsection{La recepción por los vecinos}

Respecto al reconocimiento de Brasil como una potencia —en tanto líder - regional por sus vecinos, el panorama no ha sido uniforme. Por un lado, tanto Argentina —el histórico «balanceador» regional de Brasil— como Venezuela — equipada con una política regional propia durante la era Chávez — contestaron el proyecto regional de Brasilia. En cuanto al primero, se le atribuyó a Brasil el papel de «potencia regional» pero dentro de América Latina, donde no cuenta con la misma preponderancia estructural al incluir a México, un socio de Buenos Aires en su política de balanceo (Wehner, 2015). Así también, Argentina, junto con México y Colombia, integra el foro Uniting for Consensus que se opone al ingreso del G4 ${ }^{43}$ como miembros permanentes del Consejo de Seguridad de las Naciones Unidas. Además, se interpretó al apoyo argentino a favor del ingreso de Venezuela al Mercosur como otra muestra de su balanceo frente al pivote. Respecto a Caracas, mientras que el chavismo consideró a Brasil como un socio estratégico en la región, no solamente contó con un proyecto regional propio (de proyección latinoamericana-caribeña), sino que además ha tenido, contrariamente al pragmatismo brasileño, una proyección externa abiertamente antihegemónica, originando discrepancias entre ambos vecinos. En palabras de Chávez, durante la Cumbre de América Latina y el Caribe en 2008: «Sin duda Brasil ejerce un liderazgo importante. Pero no se trata de que haya un líder en la región. Se trata de un conjunto de liderazgos» (Aporrea, 17 de diciembre de 2008).

Mientras tanto, tanto Chile (los gobiernos de la Concertación y de Sebastián Piñera) como el Perú (desde la presidencia de Alejandro Toledo hasta la candidatura electoral de Ollanta Humala) han dado muestras de reconocimiento al liderazgo brasileño. Así, por ejemplo, en 2006, Alan García Pérez eligió a Brasil para su primera visita al exterior como presidente electo y declararía: «No tenemos ningún recelo de una hegemonía constructiva de Brasil, que tiene un papel esencial como país promotor de la unión sudamericana» (La República, 13 de junio de 2006). Mientras tanto, el presidente Pińera postuló lo siguiente en 2011: «nosotros reconocemos [el liderazgo regional de Brasil] como un hecho, y esperamos que Brasil pueda representar cada vez con más fuerza no solo su propia voz sino que también juntos representaremos la voz de América Latina en este mundo nuevo que está emergiendo» (Moreno, 6

\footnotetext{
43 Alemania, Brasil, India y Japón
} 
de marzo 2011). Por otro lado, es sabido que los integrantes sudamericanos de la Alianza del Pacífico — vista por algunos como contrario al espíritu del regionalismo sudamericano- cuentan con una política exterior más orientada hacia las potencias extrarregionales y la macrorregión de la Cuenca del Pacífico, que a Brasilia.

Finalmente, tanto Bolivia, Ecuador como Uruguay criticaron la escasa disposición de Brasilia para proveer ciertos bienes regionales a cambio de dicho «seguimiento». En este sentido, las siguientes palabras expresadas en 2006 por la senadora oficialista de Uruguay, Constanza Moreira, hablaron por sí solas:

Con respecto a Brasil, creo que sigue siendo un gigante ensimismado, más allá de que la cúpula de la política exterior siga con la idea de explotar su rol como potencia mundial. Me parece que Brasil está orientado a eso, a favorecer la relación con las potencias mundiales emergentes de segundo orden. A la integración latinoamericana, Brasil la ve como algo instrumental a los efectos de eso. Con ese liderazgo, la cuestión está complicada. Brasil no está dispuesto a pagar ningún costo doméstico por la integración latinoamericana. (Rosenblatt, 2006)

Además, tal como había sucedido en la década de 1970, en los vecinos menores del Cono Sur regresaría la narrativa sobre un imperialismo brasileño en la región, siendo Raúl Zibechi probablemente el portavoz más conocido de esta lectura ${ }^{44}$. Finalmente, entre otras muestras de "descontento" frente a la presunta falta de compromiso regional por parte de Brasil, Montevideo presentaría un candidato propio (apoyado por Buenos Aires) para la secretaría general de la OMC en $2005^{45}$ y, durante varios años, mencionó la posibilidad de negociar un acuerdo comercial (bilateral) con Estados Unidos. Por todo ello, el título significativo de un artículo influyente, escrito por Malamud (2011), sobre el performance de Brasil en cuanto potencia: «A leader without followers?».

Por último, a nivel de opinión pública en la región, según los Latinobarómetros de los años 2009, 2010 y 2011, Brasil claramente sobresalió como el "país de mayor liderazgo en la región» con un $20 \%$ en el último año, frente a un 10\% para Estados Unidos y otro $10 \%$ para Venezuela. Mientras que una mayoría de uruguayos (54\%) y argentinos (52\%) compartiera esta percepción, solo lo hizo un 25\% de los propios brasileños y un $15 \%$ de ecuatorianos y venezolanos (Corporación Latinobarómetro, 2011).

\footnotetext{
${ }^{44} \mathrm{El}$ autor uruguayo es conocido por su libro Brasil potencia. Entre la integración regional y un nuevo imperialismo (2012).

45 Ocho años después, Brasil logró enviar a Roberto Azevêdo como nuevo secretario general de la OMC, esta vez con el apoyo de casi todos los vecinos sudamericanos, siendo el Perú el único de la región en apoyar al candidato mexicano, Herminio Blanco.
} 
En cuanto a Turquía, el reconocimiento como potencia regional ha sido muy dependiente de la región en cuestión. Mientras que en los Balcanes figura como potencia secundaria (luego de la UE/Alemania), en el MONA constituye una de varias potencias regionales. Como tal, la renovada proyección regional de Turquía ha sido bienvenida y reconocida por varios países — principalmente menores- en ambas regiones: Albania, Bosnia, Kosovo y Macedonia del Norte en los Balcanes ${ }^{46}$ y, actualmente, Catar en el MONA. A su vez, hasta el 2011, la histórica potencia regional del mundo árabe, Egipto, evaluó el acercamiento turco hacia la región con buenos ojos al coincidir con sus políticas de balanceo frente al rival Irán, aunque sin reconocer explícitamente al país como «potencia regional» (Abou-El-Fadl, 2012). De la misma manera, el régimen de Bashar al-Asad en Siria consideró a Turquía como un país no solo capaz de mediar en los conflictos pendientes de la región, sino además de mitigar la gran dependencia mostrada por Damasco frente a Teherán (Abou-El-Fadl, 2012). Finalmente, durante la primera etapa de la Primavera Árabe, tanto el gobierno egipcio de Mohamed Mursi como los gobiernos del movimiento Ennahda en Túnez reconocieron explícitamente a Ankara como su ejemplo y principal aliado en la región ${ }^{47}$. Cabe mencionar que Turquía, a raíz del apoyo obtenido en su región, logró ocupar el cargo de secretario general de la Organización Islámica de Cooperación de 2004 hasta 2013.

Por otro lado, de acuerdo con lo que reflejan las diversas encuestas organizadas en el Medio Oriente durante el periodo, Turquía ha sido percibida como una potencia en la región. Por ejemplo, según una encuesta de Tesev y la FES de 2009, un 79\% de los entrevistados en siete países árabes opinó que Turquía debía jugar el rol de mediador en el conflicto palestino-israelí (desde un 76\% en Arabia Saudita hasta un 89\% de palestinos), un $77 \%$ defendió un papel más importante en general en el mundo árabe y un $76 \%$ que el país podía tener un impacto positivo en la región (Akgün, Perçinoğlu y Senyücel, 2009). Según el mismo centro, para 2010, no menos de 79\% de los entrevistados tenía una percepción positiva sobre Turquía (dicha cifra luego bajó a 69\% en 2012 y 59\% en 2013), figurando así en el primer puesto antes de los Emiratos Árabés Unidos (70\%) y Arabia Saudita (64\%) (Akgün y Senyücel, 2013). Luego, a la pregunta qué país figuraba como potencia regional del MONA en 2012, Turquía lo era a nivel político, como primer país, para un 12\% de entrevistados (antes de Irán y Arabia Saudita, ambos con 9\%) y a nivel económico para un 14\%, luego de Arabia Saudita (29\%) (Akgün y Senyücel, 2012).

\footnotetext{
${ }^{46}$ Los tres primeros países además mantienen grandes vínculos culturales con Turquía, mientras que Macedonia del Norte encuentra en Ankara un socio en el conflicto que tiene con Grecia.

${ }^{47}$ Así, Rachid Ghannouchi, líder de Ennahda, describió al AKP como «the best model I can think of» (Herzog, 2014, p. 51).
} 


\subsubsection{La recepción global}

Al menos en el discurso oficial, tanto Estados Unidos como la Unión Europea tildaron a Brasil como una potencia regional y/o emergente. Así, por ejemplo, en 2005 Condoleeza Rice describió al país sudamericano como «emerging as a global power [...] a great [partner for the United States] for the future» y se refirió explícitamente a su capacidad para liderar "the way forward for all of Latin America» (2005). En el mismo año, precisamente durante una visita a Brasilia, George W. Bush nombró al país sudamericano un «socio regional», distanciándose de cierta manera de su política inicial de no reconocimiento de potencias regionales emergentes (Marra, 2008). No obstante, no pocos interpretaron esta política según los postulados de la doctrina Nixon, es decir: el reconocimiento por parte de Washington de potencias regionales aliadas a fin de "compartir responsabilidades internacionales» —y el costo que estas generan- en algunas regiones cruciales del mundo ${ }^{48}$. Además, al parecer, Brasil ha sido considerado como una especie de «baluarte» capaz de neutralizar, o al menos moderar, las posturas revisionistas de algunos países, sobre todo los del eje bolivariano. Finalmente, si el Departamento de Estado claramente respalda el ingreso de India como miembro permanente en el Consejo de Seguridad, no ha mostrado el mismo entusiasmo en cuanto a Brasil.

A su vez, la Comisión Europea se expresó en los siguientes términos sobre el rol de Brasil en la región y el mundo, antes de establecer una asociación estratégica con el país ${ }^{49}$ :

The time has come to look at Brazil as a strategic partner as well as a major Latin American economic actor and regional leader... Its emerging economic and political role brings new responsibilities for Brazil as a global leader. [...] A quasi-continent in its own right, Brazil's demographic weight and economic development make it a natural leader in South America and a key player in Latin America. (Comisión Europea, 2007, p. 1)

Además, Francia y el Reino Unido generalmente son considerados como los miembros del P5 más proclives a reformar el Consejo de Seguridad y, como co-integrante del G4, también Alemania suscribe la aspiración brasileña para integrar la principal instancia de la ONU.

Por otro lado, tanto Rusia como China han mantenido un discurso más cauteloso, evitando el término de "potencia regional» al referirse a Brasil. En cuanto a la reforma del Consejo de Seguridad, Moscú —en palabras del canciller Lavrov—

\footnotetext{
${ }^{48}$ Nixon declaró en 1971 que «[a]s Brazil goes, so will go the rest of that Latin American Continent».

49 La Unión Europea cuenta con diez socios estratégicos, siendo Brasil (2007) y México (2008) los únicos dos países de América Latina.
} 
respalda el ingreso de Brasil e India a dicho organismo, mientras que Beijing solo afirma «respetar las aspiraciones» de ambos Estados para jugar un papel más importante en la ONU (Pesnya, 15 de agosto de 2015). Las dos potencias eurasiáticas sí mantienen un diálogo político fluido con Brasil, tanto de modo bilateral, como en el marco de instancias internacionales, siendo el BRICS el más importante (Stuenkel, 2016). Brasil además fue el primer país latinoamericano en suscribir un acuerdo de Asociación Estratégica con China en 1993.

En cuanto a Turquía, al menos hasta fines del año 2009, más que como potencia regional, Washington se refirió al país como un socio importante, un Estado pivote (de acuerdo con los postulados de Huntington y Brzezinski) y, además, un modelo para la región. En este sentido, cuando George W. Bush lanza la «Iniciativa para el Medio Oriente Ampliado y el Norte de África» (o BMEI) durante una cumbre del G8 en 2004; el presidente dio a entender que una democratización de la región necesariamente pasaría por la llegada al poder de movimientos islamistas moderados (o, en palabras de Paul Wolfowitz: «una reforma islámica»), siendo el AKP el ejemplo de esta tendencia (Eksi, 2016). Así también su sucesor, Barack Obama, tildó a Turquía como líder y modelo:

The United States welcomes Turkey's leadership on the world stage to address a range of challenges that we are stronger confronting together. Our leaders meet often and work as partners on topics such as encouraging a political transition in Syria, promoting Middle East Peace, supporting economic reform and growth throughout the Middle East/North Africa region, advancing a comprehensive settlement in Cyprus, normalizing relations with Armenia, and encouraging the highest standards for human rights, democracy, and governance at home and abroad. (The White House, 2013)

De la misma manera, en una Resolución del Parlamento Europeo de 2012 se reconoce el carácter multirregional de Turquía, así como su papel en el vecindario:

[The EP] [s] tresses Turkey's strategic role, politically and geographically, for the foreign policy of the European Union and its neighborhood policy; emphasizes Turkey's role as an important regional player in the Middle East, the Western Balkans, Afghanistan/ Pakistan, the Southern Caucasus, Central Asia and the Horn of Africa and Turkey's role as a source of inspiration for democratizing Arab States in significant policy areas covering political and economic reform and institution building; expresses its support for the efforts made by Turkey to contribute to the furthering of high level dialogue and cooperation (Parlamento Europeo, 2012).

Sin embargo, a partir de 2010, en Occidente empezó a surgir un debate sobre el cambio de eje (shift of axes $^{50}$ ) en Turquía, es decir, la supuesta tendencia en Ankara

\footnotetext{
50 Otros analistas, principalmente estadounidenses, en vez de referirse al «cambio de ejes» aludieron al debate en términos de losing Turkey, muy similar al debate sobre la "pérdida de Rusia» a inicios del siglo.
} 
de distanciamiento de los tradicionales socios del Occidente a favor de una política más orientada hacia el Medio Oriente, de carácter sectarista (pro-sunita) y además, a nivel doméstico, crecientemente autoritario. En la misma línea, los medios de comunicación masivos claramente cambiaron su perspectiva sobre el papel regional de Turquía. Así, por ejemplo, la revista The Economist (8 de agosto de 2014) escribió lo siguiente sobre el país en 2014:

Until recently Turkey, a NATO member that is in membership talks with the EU has hailed as a shining example of a Muslim country where Islam and democracy can co-exist. But a mix of hubris, pro-Sunni sectarism and bad judgment on the part of the Islam-inspired Justice and Development (AK) Party, has drained the country of its soft power.

Como un reflejo de esta presunta tendencia, Turquía logró obtener en 2008 el apoyo de 151 Estados para ingresar como miembro no permanente al Consejo de Seguridad para el periodo 2009-2010, por primera vez desde 1951. No obstante, cuando nuevamente se presentó en 2014 no ingresó y obtuvo el apoyo de apenas 60 países.

\section{5. ¿El declive de ambas potencias regionales?}

Si bien no constituye el objetivo principal de la presente investigación, en este último acápite se tratará de explicar el declive de ambos países respecto a su proyección regional, al enfatizar en los cambios de la variable estructural (recursos de poder), la creciente divergencia entre las expectativas de los vecinos y el performance real de las potencias regionales en cuestión, y el papel de las grandes potencias en este proceso.

A nivel estructural, Brasil claramente perdió fuerza como potencia al entrar en una profunda crisis sociopolítica y económica a partir de 2013. Así, meses antes de los Juegos Olímpicos — que suponían una afirmación de su estatus de global player de Brasil- empezó la ola de manifestaciones contra la clase política y algunas políticas gubernamentales. Un año después inició la recesión económica, resultando en tasas de crecimiento de -3,77\% y -3,60\% para 2015 y 2016 (FMI, 2018). De esta manera, si la presidenta Rousseff en 2011 aún proyectaba que la economía brasileña estaba a punto de sobrepasar la francesa, la quinta en su momento, caería a la novena posición en 2015, detrás de Italia. En el mismo periodo, además, salieron a la luz dos casos de mega-corrupción (el Petrolão y el Lava Jato), ambos con serias implicancias para toda Sudamérica. Finalmente, a mediados de 2016, Rousseff, fue destituida mediante un impeachment y, a inicios de 2018, el nuevamente candidato presidencial, Lula da Silva, encarcelado. Todo ello, obviamente, tuvo un gran impacto en el poder blando brasileño, muy tangible en la región - y el mundo- durante la década anterior. La asunción en 2019 del ultraderechista Jair Bolsonaro como presidente tampoco contribuye con una mejora en este ámbito. 
Por otro lado, con anterioridad a dicha crisis, varios analistas ya habían cuestionado el proyecto regional de Brasil. Para empezar, con la propia salida del presidente Lula da Silva — un líder comprometido con la proyección internacional de su país y además popular en la región—, Brasilia empezaría a dar muestras de una «normalización» de su política exterior con un perfil notablemente más pragmático, un papel reducido para la presidenta y, sobre todo, mayor énfasis en la emergencia global que en asuntos regionales. En segundo lugar, a fines de la década pasada, ya se presenció un cierto deterioro en la imagen de Brasil ante la combinación de la creciente participación de empresas brasileñas en la extracción de recursos naturales en zonas vulnerables de varios países vecinos; el predomino de un enfoque unilateral de Brasil, más allá de sus vecinos, en cuestiones globales y, sobre todo; la escasa disposición de Brasilia para asumir el rol de paymaster y mediador en algunos temas cruciales para la región (como la crisis política paraguaya de 2012, el proceso de paz en Colombia o la crisis venezolana).

A nivel de las potencias, a raíz de la cercanía geográfica y la histórica hegemonía ejercida por Washington en el hemisferio occidental, la relación con Estados Unidos ha sido crucial para Brasil en su proyección como potencia emergente y regional. En este sentido, pese al discurso reconciliador y reconocedor del nuevo rol de Brasil en el mundo por parte de Washington, y el brasileño enfocado en un «acomodo geopolítico» hacia Estados Unidos ${ }^{51}$, la relación entre ambos países durante las últimas décadas no ha sido exenta de ambigüedades. Por un lado, Estados Unidos vio con buenos ojos la postura moderada de una administración de centroizquierda, tanto en asuntos internos (la macroeconomía, por ejemplo), como a nivel de política exterior, cuando Lula da Silva logró «neutralizar» algunas de las propuestas más radicales provenientes de países como Venezuela o Bolivia. Así también el rol de mediador que Brasil jugó durante la década pasada. No obstante, la búsqueda de autonomía mostrada por parte de Brasil —y apoyado por algunos vecinos-, en temas comerciales (por ejemplo, la oposición contra el proyecto ALCA) y políticos (entre otros, la resistencia contra la instalación de nuevas bases estadounidenses) claramente fue recibida con escepticismo en Washington, tal como lo demuestran algunas declaraciones de altos funcionarios. Así, por ejemplo, en un testimonio ante el Senado estadounidense en 2011, el Director de Inteligencia Nacional, James Clapper, declaró lo siguiente:

Brazil's economic success and political stability have set it on a path of regional leadership. Brasilia is likely to continue to use this influence to emphasize UNASUR as the

\footnotetext{
${ }^{51}$ Desde la década de 1990 y, aún más, durante la presidencia de Lula da Silva, ha predominado la visión de que la consolidación del estatus de Brasil como potencia (sea regional, sea emergente) también resulta de su entendimiento con Estados Unidos (Lima y Hirst, 2006).
} 
premier security and conflict resolution mechanism in the region at the expense of the OAS and of bilateral cooperation with the United States. It also will seek to leverage the organization to present a common front against Washington on regional political and security issues ${ }^{52}$. (Committee on Armed Services. United States Senate, 2011)

De la misma manera, en uno de los wikileaks, el ex embajador estadounidense en el Perú, Curtis Struble, advertiría en 2005 que Estados Unidos estaba inmerso en un concurso «no declarado» con Brasil por la influencia política sobre el Perú. Aunque, según el embajador, en aquel ańo, en la mayoría de los temas Estados Unidos salió ganando, "el gobierno de Brasil [estaba] todavía muy en el juego» (La República, 13 de agosto 2011).

En cuanto a Turquía, si bien ha perdido el dinamismo económico mostrado durante la década pasada, el ámbito donde más ha perdido terreno fue en el poder blando. En este sentido, las elecciones de 2011 resultaron cruciales al inaugurar una creciente tendencia autoritaria - y de desecularización — por parte del oficialismo. Esto quedó manifiesta durante la represión por la policía de las protestas en el Parque Gezi a mediados de 2013 — un evento denominado como la «Primavera Turca»—y, posteriormente, luego del fracasado golpe tres ańos después contra, el ahora presidente, Erdoğan.

Del mismo modo, la Primavera Árabe, considerada inicialmente como una gran oportunidad para demostrar el rol de Turquía como modelo para la región, terminó demostrando las debilidades de la política regional de Ankara. Si, inicialmente, Erdoğan y Davutoğlu se mostrarían solidarios con los manifestantes en las calles de Túnez y Egipto, no exhibieron el mismo entusiasmo hacia la oposición contra Muamar el Gadafi, al tomar en consideración la presencia de varias inversiones turcas importantes en territorio libio. Así también, hasta 2011, Turquía defendería una política regional basada en el beneficio mutuo y la afinidad cultural y religiosa con los vecinos, que además vino acorde con los principios westfalianos de no injerencia y no intervención. Aparentemente, Ankara esperaba que la creciente interdependencia entre el país y los vecinos pudiera contribuir con la democratización de los últimos (Öniş, 2012). Todo ello cambiaría con el inicio de la Primavera Árabe, cuando Turquía empezó a apoyar abiertamente a diversos movimientos y partidos derivados de la histórica Hermandad Musulmana, en su afán de exportar el modelo «AKP»y derrocar los regímenes seculares y nacionalistas (árabes) del MONA. Además, a los pocos meses después de haberse iniciado la guerra civil siria, Ankara no solo empezó a intervenir directamente en dicho vecino — poco tiempo antes considerado como un ejemplo del paradigma de «cero problemas»—, sino además a proyectar una política

\footnotetext{
52 Véase: http://www.gpo.gov/fdsys/pkg/CHRG-112shrg71354/html/CHRG-112shrg 71354.htm
} 
crecientemente sectarista (prosunita). De esta manera, Ankara se fue alejando de otro vecino crucial, la República Islámica de Irán, convirtiéndose, temporalmente, en aliado de Arabia Saudita y los Emiratos Árabes Unidos, antiejemplos de democracias en la región. A su vez, este efímero «eje sunita» pasó a fragmentarse con la llegada al poder de Mohamed Mursi, líder de la histórica Hermandad Musulmana egipcia, aliado de Turquía pero considerado como un competidor ideológico del wahabismo en el Golfo. Por ello, en palabras de Öniş y Kutlay (2017):

Ankara's overambitious desire to position itself as an «order-setting regional power» and transform the region in its mirror image led to Turkey's over-involvement in the domestic affairs of key Arab Spring countries, especially in the context of the ongoing war in Syria and the domestic turmoil in Egypt. The unintended outcome was the growing perception of Turkey as a country that increasingly contributed to instability in the region by taking an active part in sectarian conflicts that appeared to contrast sharply with its previous positive image of a benign regional power and honest broker. (p. 176)

Finalmente, luego la destitución de Mursi (2013), la crisis de Ennahda en Túnez (2014), el creciente empoderamiento de los kurdos (con el apoyo del Occidente) frente al Estado Islámico (2015-2016) y sin embajadores en El Cairo, Tel Aviv o Damasco, Ankara había perdido gran parte de su credibilidad en la región y numerosos aliados (solo mantuvo buenas relaciones con Hamas, diversos brazos nacionales de la Hermandad Musulmana y Catar). Para 2013, el propio AKP empezó a describir la situación de Turquía en su región como de «soledad preciosa» y algunos académicos, en alusión al paradigma del central country optaron por la idea del front country; un país con una proyección regional basada exclusivamente en el interés nacional y el poder duro.

Cabe mencionar que, más allá de la ambigüedad y los errores de cálculo, la crisis regional también terminó demostrando la falta de recursos de Turquía para poder perfilarse y cumplir con el estatus de potencia regional. En este sentido, un documento confidencial del gobierno estadounidense describió a la política exterior turca como «driven by Rolls Royce ambitions but Rover resources» (Herzog, 2014, p. 48). Es decir, el conflicto sirio no solo selló el colapso de la política regional de Ankara hacia el MONA, enfocada en la diplomacia pública y el poder blando, sino que además le quitó valiosos recursos para seguir con el proyecto de emergencia global. Según Eksi (2016):

Turkey [in 2016] was no more a country dealing with global policies particularly including the Middle East but a country only dealing with the border issues with Syria. In this sense, the Syrian crisis did not only bring the Middle East policy of Turkey to an end but also caused its withdrawal in the global and regional policies (p. 94).

Respecto a las grandes potencias, tal como se mencionó anteriormente, tanto Estados Unidos como los países europeos inicialmente (al menos hasta 2009) tildaron a Turquía como su nexo con el MONA y, además, un modelo para la región. No obstante, aún 
más allá de la creciente tendencia autoritaria del gobierno de Erdoğan, la búsqueda de autonomía por parte de Ankara frente a la OTAN (el así llamado «cambio de eje»), acompañada por una política no solo ambigua, sino además frecuentemente divergente con los intereses del Occidente, resultó en una profunda crisis, aún no resuelta, entre Turquía y sus históricos socios occidentales. Tanto la reticencia estadounidense a perder a un miembro tan estratégico en su principal alianza militar y la dependencia europea frente a Turquía por varias cuestiones —entre otras: la migración-, hicieron que aún no se haya llegado a una ruptura abierta entre ambos. En este sentido, al parecer, Occidente solo se mostró dispuesto a aceptar un creciente rol para Turquía en regiones tan estratégicas como el MONA y los Balcanes, siempre y cuando estuviera de acuerdo con sus respectivos intereses. Nuevamente en palabras de Eksi (2016):

Turkey lost the US support owing to a delusive ambition for the sake of becoming the leader of Muslim world and so JDP government tended to redesign its Middle Eastern policy far beyond its capacity and instruments. The U.S. opened up a space to Turkey in the region within the scope of moderate Islam project through BOP [el «BMEI»] but withdrew the support in the vicinity of Arab Spring process, particularly with respect to differing interests about Syrian crisis, when JDP elites tended towards utilizing this space to become regional leader and leader of the Muslim world instead of a model country in the region. (p. 99)

Por esta misma razón, la proyección regional de Turquía en los Balcanes no ha sufrido la misma suerte que en el MONA por haber estado más alineada con los intereses tanto de Estados Unidos, como - y sobre todo- de la Unión Europea.

Finalmente, en lo que concierne a Rusia, luego de la crisis de 2015 entre Ankara y Moscú, los dos países empezaron a retomar la agenda bilateral basada en la interdependencia comercial-energética y mutuamente conveniente ante sus fricciones con el Occidente. Además, ambos países participan, juntos con Irán, en el proceso de Astana sobre el futuro político de Siria, aunque claramente con una ventaja estratégica y de iniciativa a favor de Moscú.

\section{Conclusiones}

Tanto Brasilia como Ankara realizaron un notable ejercicio de acercamiento hacia sus respectivos vecinos durante la década pasada. Acompañada por una expansión de las inversiones y las exportaciones intrarregionales, dicha estrategia regional se basó principalmente en la proyección de poder blando, un liderazgo político del tipo transformacional y altamente proactivo en política exterior y, además, una búsqueda de autonomía frente a las grandes potencias, principalmente la hegemónica. Como tal, la asunción en el vecindario de ciertos roles tales como mediador, paymaster o líder intelectual, vinculados al perfil de potencia regional, formó parte de 
la aspiración de ambas potencias medias para emerger en la jerarquía global. En este sentido, tanto Ankara como Brasilia consideraron que su emergencia global estuvo precondicionada por sus relaciones con los vecinos - $y$, más concreto, la legitimidad obtenida por ellos-, más allá de su creciente participación en el multilateralismo global y la búsqueda de reconocimiento por parte de las grandes potencias. Esta «tesis del trampolín» además ha sido replicada por la academia al conectar los conceptos de potencias medias (de segunda generación), las emergentes y las regionales.

Luego de una revisión de la literatura sobre los tres tipos de potencias mencionados, la presente investigación intentó realizar una evaluación para Brasil y Turquía de las cuatro dimensiones propias de una potencia regional: su preponderancia estructural en la región, la autopercepción de carácter excepcionalista en cuanto pivote de la región, un repertorio de estrategias de política exterior implementadas a fin de afirmarse como potencia regional, y el grado de reconocimiento obtenido, sea por los vecinos, sea por las potencias extrarregionales. Además, se trató de determinar por qué tanto Brasil como Turquía han perdido fuerza en su proyección como potencia regional durante la presente década, enfatizando la pérdida de ciertos recursos de poder en este periodo (principalmente poder blando y/o económico), la creciente divergencia entre las expectativas entre vecinos y la performance de las PR y la postura de las grandes potencias.

A partir de lo investigado se ha llegado a las siguientes conclusiones:

Uno. La proyección como potencia regional para un Estado acarrea la generación de ciertas expectativas entre los vecinos respecto a los diversos roles asociados a dicho concepto. Así, el vecindario no solamente espera recompensas económicas (por ejemplo, mediante acuerdos de preferencias comerciales o la inversión directa de sus recursos) a cambio de su respectivo seguimiento, sino además la formulación de una agenda que responda a los intereses de la región entera - y no solamente de la potencia en cuestión-, una gestión imparcial y eficaz de mediación, y hasta un grado de representación de la región por parte de la potencia regional en espacios globales. Cumplir con esta provisión de bienes regionales implica un costo para dichas potencias, tanto en términos económicos, como políticos (a nivel de autonomía, principalmente). Al pertenecer aún a la semi-periferia del sistema global, las PR no siempre disponen de dichos recursos, también muy necesarios para el desarrollo interno, así como para invertir en la emergencia global, generando así una divergencia entre las expectativas de los vecinos y el performance de las potencias regionales. Por otro lado, pese a la creciente interdependencia económica mostrada en los últimos años por las potencias regionales respecto a sus vecinos, en comparación con «regiones céntricas» como Europa, América del Norte y hasta Asia Oriental, con flujos intrarregionales más altos, las $\mathrm{PR}$ aún no pueden presentarse como una alternativa real frente a los mercados de las potencias extrarregionales. 
Dos. Buscar simultáneamente una regionalización de la política exterior y una emergencia en la jerarquía global puede conllevar tensiones entre ambas misiones. Se ha observado que ninguna de las potencias regionales estudiadas realmente se mostró dispuesta a ser el «representante regional» en foros globales, al parecer por la contradicción que puede surgir entre la búsqueda de autonomía, un elemento clave de las potencias emergentes, por un lado y, por otro lado, un mayor compromiso con los vecinos. Además, la propia preponderancia estructural aunada al recuerdo de ciertos episodios de la historia regional (por ejemplo, conflictos interestatales, el intervencionismo histórico o un legado imperial de las PR), puede originar la percepción de una potencia que busca delimitar su zona de influencia o imperio a costo de los vecinos. Finalmente, las PR pueden encontrar resistencia en la región, sobre todo por parte de las PR secundarias que suelen liderar políticas de soft balancing frente a las primeras. Por lo tanto, si bien es cierto que las potencias regionales pueden funcionar como nexos entre su sistema regional y el global, casos como Brasil y Turquía han demostrado que la región puede reducir, en lugar de incrementar la proyección de poder a nivel global.

Tres. Más allá del declive en el poder blando y económico y el creciente mismatch entre expectativas y la provisión real de bienes regionales por parte de Brasil y Turquía en sus regiones, también observamos que resulta muy complejo encontrar un equilibrio entre la acumulación de cierta autonomía en cuanto potencia emergente y la búsqueda de reconocimiento por parte de las potencias extrarregionales. Mientras que Washington (y Bruselas) pueden mostrarse dispuestos a reconocer el estatus de potencia regional/emergente a fin de compartir el costo de una presencia geopolítica en algunas regiones clave del mundo, dicho reconocimiento además queda sujeto al grado de coincidencia política y/o la posibilidad de cooptar a dichos Estados en función de sus intereses. En cambio, cualquier propuesta de reforma proveniente de los emergentes y capaz de afectar o cuestionar el estatus de las potencias establecidas, tiende a ser clasificada como "antisistémica» (o «revisionista») y a generar mecanismos de contención. En cuanto a China y Rusia, ellas mismas aún (re-) emergiendo, en la práctica parecen mostrarse más dispuestos a reconocer los roles de PE/PR, siempre y cuando no genere un impacto negativo para su propia emergencia global y proyección regional.

Cuatro. Brasil y Turquía han demostrado que convertirse en una potencia regional no solo resulta costoso, sino que además implica un ejercicio diplomático sumamente delicado. Como tal, la región suele representar más un obstáculo que una bisagra para obtener el reconocimiento global como potencia. Además, tanto ejemplos históricos (Gran Bretaña o Estados Unidos) como actuales (India), demuestran que, equipados con una base de recursos de poder duro y una actitud pragmática hacia las potencias consolidadas, la emergencia global puede ser exitosa, pese a los vecinos. 


\section{Referencias bibliográficas}

Abou-El-Fadl, R. (2012). Arab perceptions of Contemporary Turkish Foreign Policy: Cautious Engagement and the Question of Independence. En K. Öktem, A. Kadıoglu y M. Karli. Another Empire? A decade of Turkey's Foreign Policy under the Justice and Development Party (pp. 231-257). Estambul: Istanbul Bilgi University Press.

Acharya, A. (2018). The End of American World Order. Cambridge: Polity.

Akgün, M., Perçinoğlu, G. y Senyücel, S. (2009). The perception of Turkey in the Middle East. Estambul: Tesev Publications.

Akgün, M. y Senyücel, S. (2012). The perception of Turkey in the Middle East 2012. Estambul: Tesev Publications.

Akgün, M. y Senyücel, S. (2013). The perception of Turkey in the Middle East 2013. Estambul: Tesev Foreign Policy Programme.

Almeida, P. (2010). Never before Seen in Brazil: Luís Inácio da Silva's grand diplomacy. Revista Brasileira de Politica Internacional, 53(2), 160-177. https://doi.org/10.1590/ S0034-73292010000200009

Altunışı, M. (2014). Geopolitical representation of Turkey's cuspness. Discourse and practice. En M. Herzog y P. Robins, The Role, Position and Agency of Cusp States in International Relations (pp. 25-41). Nueva York: Routledge. https://doi. org/10.4324/9781315768472-2

Amorim, C. (2010). Brazilian Foreign Policy under President Lula (2003-2010): An overview. Revista Brasileira de Politica Internacional, 53, 214-240. https://doi.org/10.1590/ S0034-73292010000300013

Aporrea (17 de diciembre de 2008). Presidente Chávez: «El liderazgo de Brasil es importante, pero no el único». Recuperado de https:/www.aporrea.org/actualidad/n125702.html

Burges, S. (2009). Brazilian Foreign Policy after the Cold War. Gainesville: University Press of Florida. https://doi.org/10.5744/florida/9780813033334.001.0001

Buzan, B. y Wrever, O. (2004). Regions and Powers. The Structure of International Security. Cambridge: Cambridge University Press.

Cagaptay,S.(16deagostode2013). TheMuslimBrotherhood'sFallLandsTurkeyanUnexpectedAlly: Kurds. The Atlantic. Recuperadodehttps:/www.washingtoninstitute.org/policy-analysis/ view/the-muslim-brotherhoods-fall-lands-turkey-an-unexpected-ally-kurds

Comisión Europea (2007). Communication from the Commission to the European Parliamentand the Council-Towards an EU-BrazilStrategic Partnership. (COM/2007/0281). Recuperado de https://eur-lex.europa.eu/legal-content/EN/ALL/?uri=CELEX\%3A52007DC0281

Committee on Armed Services. United States Senate. (2011). The current and future worldwide threats to the National Security of the United States. Senate Hearing 112-159. Washington DC: U.S. Government Printing Office. Recuperado de https://www. govinfo.gov/content/pkg/CHRG-112shrg71354/html/CHRG-112shrg71354.htm

Cooper, D. (2011). Challenging Contemporary Notions of Middle Power Influence: Implications of the Proliferation Security Initiative for 'Middle Power Theory'. Foreign Policy Analysis, 7, 317-336. https://doi.org/10.1111/j.1743-8594.2011.00140.x 
Cooper, D. (2013). Somewhere between Great and Small: Disentangling the Conceptual Jumble of Middle, Regional, and 'Niche' Powers. The Journal of Diplomacy and International Relations, 14(2), 23-35. Recuperado de https://apps.dtic.mil/dtic/tr/ fulltext/u2/1014484.pdf

Corporación Latinobarómetro (2012). Informe 2011. Santiago: Corporación Latinobarómetro.

Couto, L. (2007). O horizonte regional do Brasil e a construção da América do Sul. Revista Brasileira de Política Internacional, 50, 159-176. https://doi.org/10.1590/ S0034-73292007000100009

Cox, R. y Sinclair, T. (1996). Approaches to World Order. Cambridge: University of Cambridge Press. https://doi.org/10.1017/CBO9780511607905

Davutoğlu, A. (Noviembre de 2011). Vision 2023: Turkey's Foreign Policy Objectives. En Goldman Sachs, Turkey Investor Conference: The road to 2023. Londres, Reino Unido. Recuperado de http://www.mfa.gov.tr/speech-entitled-_vision-2023_-turkey_sforeign-policy-objectives__-delivered-by-h_e_-ahmet-davutoglu_-minister-offoreign-af.en.mfa

Davutoglu, A. (2013). Turkey's Mediation: critical reflections from the field. Middle East Policy, 20(1), 83-90. https://doi.org/10.1111/mepo.12005

Del Arenal, C. (1983). Poder y relaciones internacionales: un análisis conceptual. Revista de Estudios Internacionales, 4(3), 501-524.

Destradi, S. (2010). Regional powers and their strategies: empire, hegemony and leadership. Review of International Studies, 36, 903-930. https://doi.org/10.1017/ S0260210510001361

Devinit (2017). Global Humanitarian Assistance Report 2017. Bristol: Development Initiatives. Recuperado de http://devinit.org/wp-content/uploads/2017/06/GHA-Report-2017Full-report.pdf

Dewitt, D. y Christie, R. (2007). Los poderes medios y la seguridad regional. En J. Tokatlian (Comp.), India, Brasil y Sudáfrica. El impacto de las nuevas potencias regionales (pp. 55-95). Buenos Aires: Zorzal.

Eksi, M. (2016). The Rise and Fall of Soft Power in Turkish Foreign Policy during JDP. Saarbrücken: Lambert Academic Publishing.

ESI (4 de diciembre de 2010). Is Turkey's Balkan policy today inspired by a postmodern vision of the world? A pre-modern one? And does it matter how we call it? Recuperado de http://www.esiweb.org/rumeliobserver/2010/12/04/ multikulti-and-the-future-of-turkish-balkan-policy

Fisher, N. (2012). 'Democratic Depth': The Missing Ingredient in Turkey's Domestic/Foreign Policy Nexus. K. Öktem, A. Kadıôlu y M. Karli, Another Empire? A decade of Turkey's Foreign Policy under the Justice and Development Party (pp. 61-75). Estambul: Istanbul Bilgi University Press.

Flemes, D. (2007). Conceptualizing Regional Power in International Relations - Lessons from the South African Case. Giga Working Paper, 53. https://doi.org/10.2139/ ssrn. 1000123 
Flemes, D. y Wojczewski, T. (2011). Contested Leadership in Comparative Perspective: Power Strategies in South Asia and South America. Asian Journal of Latin American Studies, 24(1), 1-27.

Föhrig, A. (2007). Resistencias y equilibrios regionales. En J. Tokatlian (Comp.), India, Brasil y Sudáfrica. El Impacto de las nuevas potencias regionales. Buenos Aires: Zorzal.

Fonseca, P., Paes, L. y Cunha, A. (2016). The concept of emerging power in international politics and economy. Brazilian Journal of Political Economy, 36(1), 46-69. https:// doi.org/10.1590/0101-31572016v36n01a04

Gardini, G.L. (2016). Brazil: What Rise of What Power? Bulletin of Latin American Research, 35(1), 5-19. https://doi.org/10.1111/blar.12417

Garzón Pereira, J. (2013). Hierarchical regional orders: An analytical framework. Journal of Policy Modeling, 36, 26-46. https://doi.org/10.1016/j.jpolmod.2013.10.007

Herzog, M. (2014). From cusp to hub? How Turkey tried to instrumentalize its cuspness as an aspiring multi-regional middle power. En M. Herzog y P. Robins (eds.), The Role, Position and Agency of Cusp States in International Relations (pp. 42-59). Nueva York: Routledge. https://doi.org/10.4324/9781315768472-3

Holbraad, C. (1972). El papel de las potencias medias en la política internacional. Estudios Internacionales, 5(17), 53-75. https://doi.org/10.5354/0719-3769.1972.18879

Hulse, M. (2016). Regional Powers and Leadership in Regional Institutions: Nigeria in ECOWAS and South Africa in SADC. KFG Working Paper 76. Berlín: Freie Universität Berlin / Kolleg-Forschergruppe (KFG) "The Transformative Power of Europe".

Hurrell,A.(2006).Hegemony, liberalismand globalorder: whatspaceforwould-begreatpowers? InternationalAffairs, 82(1), 1-19. https://doi.org/10.1111/j.1468-2346.2006.00512.x

Hurrell, A. (2007). One world? Many worlds? The place of regions in the study of international society. International Affairs, 83(1), 127-146. https://doi. org/10.1111/j.1468-2346.2007.00606.x

Jordaan, E. (2003). The concept of a middle power in international relations: distinguishing between emerging and traditional middle powers. Politikon, 30(2), 165-181. https:// doi.org/10.1080/0258934032000147282

Kahler, M. (2013). Rising powers and global governance: negotiating change in a resilient status quo. International Affairs, 89(3), 711-729. https://doi.org/10.1111/1468-2346.12041

Kardaş, Ș. (2013). Turkey: a Regional Power facing a Changing International System. Turkish Studies, 14(4), 637-660. https://doi.org/10.1080/14683849.2013.861111

Kugler, J. y Organski, A. (1989). The Power Transition: a Retrospective and Prospective Evaluation. En M. Midlarsky (ed.), Handbook of War Studies. Nueva York: Routledge.

La República (13 de junio de 2006). CAN tiene futuro sin Chávez. La República. Recuperado de https://larepublica.pe/politica/277751-can-tiene-futuro-sin-chavez/

Lafer, C. (2002). La identidad internacional de Brasil. Buenos Aires: Fondo de Cultura Económica.

Lemke, D. (2010). Dimensions of Hard Power: Regional Leadership and Material Capabilities. En D. Flemes (ed.), Regional Leadership in the Global System: Ideas, Interests and Strategies of Regional Powers. Farnham: Ashgate. 
Lima, M.R. y Hirst, M. (2006). Brazil as an intermediate state and regional power: action, choice and responsibilities. International Affairs, 82(1), 21-40. https://doi. org/10.1111/j.1468-2346.2006.00513.x

Malamud, A. (2011). A Leader without Followers? The Growing Divergence between the Regional and Global Performance of Brazilian Foreign Policy. Latin American Politics and Society, 53(3), 1-24. https://doi.org/10.1111/j.1548-2456.2011.00123.x

Marirrodriga, J. (3 de noviembre de 2006). Quiero una relación privilegiada con EE UU. El Pais. Recuperado de https://elpais.com/diario/2006/11/03/internacional/ 1162508402_850215.html

Marra, T. (2008). Brasil y la integración sudamericana a comienzos del siglo 21. Estudios Internacionales, 159, 97-110.

Ministerio de Relaciones Exteriores de la República de Turquía - MFA (2018a). Relations with the Balkan Region. http://www.mfa.gov.tr/relations-with-the-balkan-region.en.mfa

Ministerio de Relaciones Exteriores de la República de Turquía - MFA (2018b). Turkey's Relations with Southern Caucasus Countries. Recuperado de http://www.mfa.gov.tr/ sub.en.mfa?6bda4d8b-81a3-44d7-b8a0-5a287804f77b

Ministerio de Relaciones Exteriores de la República de Turquía - MFA (2018c). Turkey's Relations with the Arab Countries. Recuperado de http://www.mfa.gov.tr/turkey_srelations-with-the-arab-countries.en.mfa

Moreno, J. (6 de marzo de 2011). Nos hemos propuesto terminar con la pobreza extrema en cuatro ańos. El País. Recuperado de https://elpais.com/diario/2011/03/06/ domingo/1299387158_850215.html

Mitchell, D. (2016). Regional Power? Yes, but What Kind? Paper presentado en ISA Asia Pacific, Hong Kong.

Narkilar, A. (2013). Negotiating the rise of power. International Affairs, 89(3), 516-576. https://doi.org/10.1111/1468-2346.12033

National Intelligence Council - NIC (2004). Mapping the Global Future. Pittsburgh: Government Printing Office. Recuperado de http://www.futurebrief.com/ project2020.pdf

Nel, P. (2010). Redistribution and Recognition: what emerging regional powers want. Review of International Studies, 36, 951-974. https://doi.org/10.1017/S0260210510001385

Nolte, D. (2006). Potencias regionales en la politica internacional: conceptos y enfoques de análisis. GIGA Working Paper 30. Hamburgo: GIGA German Institute of Global and Area Studies / Leibniz-Institut für Globale und Regionale Studien.

Nolte, D. (2010). How to compare regional powers: analytical concepts and research topics. Review of International Studies, 36, 881-901. https://doi.org/10.1017/ S026021051000135X

Nolte, D. (2011). Regional powers and regional governance. En N. Godehardt y D. Nabers (eds.), Regional Powers and Regional Orders (pp. 49-67). Londres: Routledge.

Nye, J. (2004). Soft Power: The Means to Success in World Politics. Cambridge: Perseus Books.

Öniş, Z. (2012). Turkey and the Arab Spring: between Ethics and Self-Interest. Insight Turkey, 14(3), 45-63. https://doi.org/10.2139/ssrn.2017639 
Öniş, Z. y Kutlay. M. (2017). The dynamics of emerging middle-power influence in regional and global governance: the paradoxical case of Turkey. Australian Journal of International Affairs, 71(2), 164-183. https://doi.org/10.1080/10357718.2016.1183586

Oskececi-Taner, B. (2005). The Impact of Institutionalized Ideas in Coalition Foreign Policy Making: Turkey as an Example, 1991-2002. Foreign Policy Analysis, 1(3), 249-278. https://doi.org/10.1111/j.1743-8594.2005.00012.x

Özel, S. y Özkan, B. (2015). Illusions versus reality: Turkey's approach to the Middle East and North Africa. Policy Brief, 200, 1-7.

Parlamento Europeo (2012). European Parliament resolution on the 2011 Progress Report on Turkey (2011/2889(RSP)). Recuperado de http://www.europarl.europa.eu/sides/ getDoc.do?type $=$ MOTION\&reference $=$ B7-2012-0189\&language $=\mathrm{EN}$

Parlar, E. (2016). Conceptualizing and testing the 'emerging regional power' of Turkey in the shifting international order. Third World Quarterly, 37(8), 1425-1453. https://doi. org/10.1080/01436597.2016.1142367

Pedersen, T. (2002). Cooperative hegemony: power, ideas and institutions in regional integration. Review of International Studies, 28, 677-696. https://doi.org/10.1017/ S0260210502006770

Pesnya, V. (15 de agosto de 2015). Russia supports applications of India, Brazil for permanent membership in UN SC. Russia Beyond. Recuperado de https://www.rbth.com/ world/2015/08/15/rusia-supports-applications-of-india-brazil-for-permanent-membership-in-un-sc_390367

Prys, M. (2010). The variability of regional powers. Hamburgo: GIGA.

Robertson, J. (2017). Middle-power definitions: confusion reigns supreme. Australian Journal of International Affairs, 71(84), 355-370. https://doi.org/10.1080/1035771 8.2017.1293608

Rosenblatt, F. (2006). Charlas para armar: Kurt Weyland, Constanza Moreira y Jorge Lanzaro. Hablemos sobre populismo. Montevideo Portal. Recuperado de https://www.montevideo.com.uy/ZZZ-No-se-usa/Charlas-para-armar-KurtWeyland-Constanza-Moreira-y-Jorge-Lanzaro-uc30564

Saraiva, M.G. (2012). Procesos de integración de América del Sur y el papel de Brasil. Revista CIDOB d'afers internacional, 97-98(abril), 87-100.

Schirm, S. (2005). Führungsindikatoren und Erklärungsvariablen für die neue internationale Politik Brasiliens. Lateinamerika Analysen, 4(11), 107-130.

Schoeman, M. (2000). South Africa as an Emerging Middle Power. African Security Review, 9(3), 47-58. https://doi.org/10.1080/10246029.2000.9628050

Serfaty, S. (2011). Moving into a Post-Western World. The Washington Quarterly, 34(2), 7-23. https://doi.org/10.1080/0163660X.2011.562080

Spektor, M. (2010). Ideias de ativismo regional: a transformação das leituras brasileiras da regiāo. Revista Brasileira de Politica Internacional, 53(1), 25-44. https://doi. org/10.1590/S0034-73292010000100002

Stuenkel, O. (2016). Post-Western World. How Emerging Powers are remaking Global Order. Cambridge: Polity Press. 
The Guardian (13 de setiembre de 2011). Libya, Egypt, Syria and Middle East unrest. Recuperado de https:/www.theguardian.com/world/middle-east-live/2011/sep/13/ libya-syria-egypt-middle-east-unrest-live-updates

The White House (2013). Fact Sheet: U.S.-Turkey Partnership. Recuperado de http://www. presidency.ucsb.edu/ws/?pid=122788

Vallès, J. (2010). Ciencia política: una introducción. Barcelona: Ariel.

Van Der Westhuizen, J. (1998). South Africa's Emergence as a Middle Power. Third World Quarterly, 19(3), 435-455. https://doi.org/10.1080/01436599814334

Vigevani, J. y Favaron, G. (2008). O papel da integração regional para o Brasil: universalismo, soberania e percepção das elites. Revista Brasileira de Politica Internacional, 51(1), 5-27. https://doi.org/10.1590/S0034-73292008000100001

Walker, J. (2 de julio de 2011). The Return of the Turks as the Middle East's Kingmaker. Huffpost. Recuperado dehttps://www.huffpost.com/entry/the-return-of-the-turks-a_b_818402

Wight, M. (1978). Power Politics. Londres: Continuum.

Yilmaz, Ö. (2014). A constructivist analysis of Turkish Foreign Policy in 1980s and 2000s: The example of Turkey-U.S. relations. Dissertation im fach Politikwissenschaft. Siegen: Philosophischen Fakultät der Universität Siegen.

Young, O. (1991). Political Leadership and Regime Formation: On the Development of Institutions in International Society. International Organization, 45(3), 281-308.

Zakaria, F. (2008). The Post-American World. Nueva York: W.W. Norton \& Company. https:// doi.org/10.1111/j.1540-5842.2008.00993.x

Zibechi, R. (2012). Brasil potencia. Entre la integración regional y un nuevo imperialismo. Bogotá: Ediciones Desde Abajo.

Recibido: 30 de abril de 2019

Aprobado: 5 de agosto de 2019 